\title{
The Association of Urinary Polycyclic Aromatic Hydrocarbons Biomarkers and Markers of Inflammation, Diabetes Mellitus and Cardiovascular Disease
}

\author{
Omayma Alshaarawy \\ West Virginia University
}

Follow this and additional works at: https://researchrepository.wvu.edu/etd

\author{
Recommended Citation \\ Alshaarawy, Omayma, "The Association of Urinary Polycyclic Aromatic Hydrocarbons Biomarkers and \\ Markers of Inflammation, Diabetes Mellitus and Cardiovascular Disease" (2013). Graduate Theses, \\ Dissertations, and Problem Reports. 4946. \\ https://researchrepository.wvu.edu/etd/4946
}

This Dissertation is protected by copyright and/or related rights. It has been brought to you by the The Research Repository @ WVU with permission from the rights-holder(s). You are free to use this Dissertation in any way that is permitted by the copyright and related rights legislation that applies to your use. For other uses you must obtain permission from the rights-holder(s) directly, unless additional rights are indicated by a Creative Commons license in the record and/ or on the work itself. This Dissertation has been accepted for inclusion in WVU Graduate Theses, Dissertations, and Problem Reports collection by an authorized administrator of The Research Repository @ WVU. For more information, please contact researchrepository@mail.wvu.edu. 
The Association of Urinary Polycyclic Aromatic Hydrocarbons Biomarkers and Markers of Inflammation, Diabetes Mellitus and Cardiovascular Disease

\section{Omayma Alshaarawy}

Dissertation submitted to the School of Public Health at West Virginia University in partial fulfillment of the requirements for the degree of

Doctor of Philosophy in Epidemiology

Michael Andrew, Ph.D., Chair

Alan Ducatman, M.D, MSC

Jamal Mustafa, Ph.D.

Motao Zhu, M.D., M.S., Ph.D.

Baqiyyah Conway, MA, MPH, Ph.D.

Morgantown, West Virginia

2013

Keywords: Polycyclic Aromatic hydrocarbons; Systemic Inflammation;

Diabetes Mellitus; Cardiovascular Diseases

Copyright 2013 Omayma Alshaarawy 


\title{
ABSTRACT \\ The Association of Urinary Polycyclic Aromatic Hydrocarbons and Markers of Inflammation, Diabetes Mellitus and Cardiovascular Disease
}

Omayma Alshaarawy

\begin{abstract}
Polycyclic aromatic hydrocarbons are potent atmospheric pollutants, released into air during incomplete combustion of fuel, industrial or domestic coal, wood, cigarette smoke and other organic materials. In addition to being carcinogenic, several animal studies have reported positive associations between polycyclic aromatic hydrocarbons and inflammation, oxidative stress and the development and progression of atherosclerosis. Occupational studies have reported positive associations between polycyclic aromatic hydrocarbons and cardiovascular morbidity and mortality. Moreover, there is evidence that polycyclic aromatic hydrocarbons may cause disruption of the endocrine system. It is still not clear if lower background exposure to polycyclic aromatic hydrocarbons, independent of the adverse health effects of smoking, is associated with increased risk of inflammation, diabetes mellitus and cardiovascular disease in the general population. We examined participants from the merged National Health and Nutrition Examination Survey 2001-02, 2003-2004, and 2005-2006. Our exposures of interest were eight urinary monohydroxy polycyclic aromatic hydrocarbons and our outcomes were serum markers of systemic inflammation, including: serum C-reactive protein and total white blood cell count, diabetes mellitus and self-reported cardiovascular disease. Urinary biomarkers of the low molecular weight polycyclic aromatic hydrocarbons were found to be positively associated with serum C-reactive protein, total white blood cell count and diabetes mellitus independent of potential confounders. Levels of 1-hydroxypyrene, the urinary metabolite of the higher molecular weight PAH, pyrene, showed a less strong association with serum C-reactive protein and diabetes mellitus. The evidence on the association between polycyclic aromatic hydrocarbons and self-reported cardiovascular disease was limited, only 1-hydroxynapthalene, summed biomarkers of low molecular weight polycyclic aromatic hydrocarbons, and 1hydroxypyrene showed statistically significant positive associations with cardiovascular disease independent of potential confounders. In conclusion, this study provides evidence on the positive association of background exposure to polycyclic aromatic hydrocarbons and serum C-reactive protein, total white blood cell count, diabetes mellitus and cardiovascular disease. Further prospective studies are needed to replicate or refute our findings.
\end{abstract}




\section{DEDICATION}

This dissertation is dedicated to Mr. Omar Alshaarawy and Mrs. Magda Alhalawany, my parents to whom I owe everything. 


\section{ACKNOWLEDGMENTS}

The process of conducting research is unfeasible without the support of numerous people. I owe debt of gratitude to all those people who have made this dissertation possible and because of whom my graduate experience has been an enriching and rewarding one. I want to deeply thank my husband Dr. Hosam Elbaz, my siblings Mohamed, Hend and Mennah for all their support in my personal and professional life.

I am deeply indebted to my esteemed advisor, Dr. Michael Andrew for his encouragement, support, patience and guidance. He is an excellent scientist and mentor, and one of the most efficient persons I have ever encountered. I believe that I am quite fortunate to have had the opportunity to work with him. I would also like to thank my committee members, Dr. Alan Ducatman, Dr. Jamal Mustafa, Dr. Motao Zhu, and Dr Baqiyyah Conway for their insightful comments and valuable suggestions that helped in my research. I would also like to thank the faculty, staff and graduate students of the School of Public Health for their help and support. 


\section{TABLE OF CONTENTS}

LIST OF TABLES

vi-vii

LIST OF SYMBOLS

viii

INTRODUCTION

1

CHAPTER 1: The Association of Urinary Polycyclic Aromatic Hydrocarbon Biomarkers and Serum Inflammatory Markers.

CHAPTER 2: The Association of Urinary Polycyclic Aromatic Hydrocarbon Biomarkers and

Diabetes Mellitus.

CHAPTER 3: The Association of Urinary Polycyclic Aromatic Hydrocarbon Biomarkers and Cardiovascular Disease. 


\section{LIST OF TABLES}

\section{CHAPTER 1: The Association of Urinary Polycyclic Aromatic Hydrocarbon Biomarkers}

\section{and Serum Inflammatory Markers.}

TABLE 1: Baseline characteristics of the study population

TABLE 2: Weighted percentiles of OH-PAH (ng/g creatinine) among participants with serum CRP levels $\leq 10 \mathrm{mg} / \mathrm{L}$

TABLE 3: Weighted percentiles of OH-PAH (ng/g creatinine) among participants with total WBC count (4000-12,000cells/uL)

TABLE 4: Weighted Pearson correlations between log-transformed urinary OH-PAH (ng/g creatinine)

TABLE 5: Linear regression: Association of urinary OH-PAH (ng/g creatinine) and serum CRP $(\mathrm{mg} / \mathrm{L})$

TABLE 6: Linear regression: Association of urinary OH-PAH (ng/g creatinine) and total WBC count (cells/uL)

TABLE 7: Logistic regression: Association of urinary OH-PAH (ng/g creatinine) and serum $\mathrm{CRP} \geq 3 \mathrm{mg} / \mathrm{L}$

TABLE 8: Logistic regression: Association of urinary OH-PAH (ng/g creatinine) and total WBC count (cells/uL) at or above the 95th percentile of total WBC count distribution 26 TABLE 9: Logistic regression: Association of summed urinary biomarkers of LMW PAHs (ng/g creatinine) and serum inflammatory markers by sociodemographic characteristics 
CHAPTER 2: The Association of Urinary Polycyclic Aromatic Hydrocarbon Biomarkers and Diabetes Mellitus.

TABLE 1: Baseline characteristics of the study population $\quad 40$

TABLE 2: Weighted percentiles of OH-PAH (ng/g creatinine) among participants 41

TABLE 3: Linear regression: Association of urinary OH-PAH (ng/g creatinine) and

glycosylated hemoglobin (\%) 42

TABLE 4: Linear regression: Association of urinary OH-PAH (ng/g creatinine) and fasting

blood glucose (mg/dL)

TABLE 5: Logistic regression: Association of urinary OH-PAH (ng/g creatinine) and diabetes mellitus

TABLE 6: Logistic regression: Association of urinary OH-PAH (ng/g creatinine) and diabetes mellitus by sociodemographic characteristics

CHAPTER 3: The Association of Urinary Polycyclic Aromatic Hydrocarbon Biomarkers and Cardiovascular Disease.

TABLE 1: Baseline characteristics of the study population $\quad 58$

TABLE 2: Weighted percentiles of OH-PAH (ng/g creatinine) among participant 59

TABLE 3: Logistic regression: Association of urinary OH-PAH (ng/g creatinine) and self-

$\begin{array}{ll}\text { reported cardiovascular disease } & 60\end{array}$

TABLE 4: Logistic regression: Association of urinary OH-PAH (ng/g creatinine) and selfreported CVD by sociodemographic characteristics 


\section{LIST OF SYMBOLS}

1. PAHs: Polycyclic aromatic hydrocarbons

2. CVD: cardiovascular disease

3. CRP: C-reactive protein

4. WBC: White blood cell count

5. US: United States

6. LMW PAHs: low molecular weight polycyclic aromatic hydrocarbons

7. SHS: Second hand smoke

8. BMI: Body mass index

9. OH-PAH: Monohydroxy polycyclic aromatic hydrocarbons

10. NHANES : National Health and Nutrition Examination Survey

11. 1-NAP : 1-hydroxnapthalene

12. 2-NAP: 2-hydroxynapthalene

13. 2-FLU: 2-hydroxyfluorene

14. 3-FLU: 3- hydroxyfluorene

15. 1-PHEN: 1-hydroxyphenanthrene

16. 2-PHEN: 2- hydroxyphenanthrene

17. 3-PHEN:3- hydroxyphenanthrene

18. 1-PYR: 1-hydroxypyrene 


\section{INTRODUCTION}

\section{Rationale for studying systemic inflammation, diabetes mellitus and CVD}

Despite advances in prevention, diagnosis and treatment, cardiovascular disease (CVD) remains the number one cause of mortality in United States (US) adults.[1] Diabetes mellitus is a major risk factor for CVD [2,3] and the seventh leading cause of death in the US.[4] Systemic inflammation is considered a key risk factor for atherosclerosis and subsequent development of CVD.[5] Inflammation has been also known to associate with insulin resistance and diabetes mellitus.[6] Several studies have reported positive association between baseline elevations of the serum inflammatory marker C-reactive protein (CRP) and risk of CVD and diabetes mellitus.[7, 8] Moreover, elevation in total white blood cells (WBC) count within the normal range was found to be independently associated with CVD and diabetes mellitus.[9, 10]

\section{Environmental pollutants and systemic inflammation, diabetes mellitus and CVD}

Several studies have suggested that exposure to particulate matter present in ambient air is associated with increased CVD. Recent evidence has suggested that smaller particles and gases may pose a greater CVD risk, possibly through development of atherosclerosis.[11] In addition, studies have suggested that common environmental exposures, such as Dioxins [12] (group of halogenated aromatic hydrocarbons), affecting large sections of the population may be a determinant of diabetes risk [13, 14] as well as CVD risk.[15]

\section{Polycyclic aromatic hydrocarbons}


Polycyclic aromatic hydrocarbons (PAHs) are environmental and occupational carcinogens released into air during incomplete combustion of fuel, industrial or domestic coal, wood, and grilled or smoked food.[16, 17] Active smoking and second-hand smoke (SHS) exposure are major sources of PAHs.[18] Individuals without occupational or tobacco smoke exposure are believed to be exposed primarily to PAHs through food contaminated with PAHs.[19] The total intake of PAHs in the general population has been estimated to be 3 $\mu \mathrm{g} /$ day.[20]

Polycyclic aromatic hydrocarbons are found within the environment in either a gaseous or particulate form.[21] PAHs with the lowest molecular weight (naphthalene, fluorene, and phenanthrene) have low vapor pressures, and are found mainly in a gaseous form. Polycyclic aromatic hydrocarbons with higher molecular weights (pyrene) have higher vapor pressures and are found in a particulate form. Individuals exposed to PAHs via inhalation of the ambient air are exposed to both low molecular weight (LMW) PAHs and higher molecular weight PAHs. Humans are also exposed to the higher molecular weight PAHs through gastrointestinal absorption of contaminated food or water and/or skin absorption of PAH particles.[22]

Polycyclic aromatic hydrocarbons were found to have high affinity for lipid-rich tissues such as mammary and adipose tissues.[23] Irrespective of the route of administration, PAHs are rapidly and widely distributed in the body. Detectable levels of PAHs can be observed in most internal organs following exposure.[4] Researchers have examined PAHs directly in the blood and tissues of experimental animals. However, these methods have not been widely used for humans due to the high costs and the limited clinical significance of testing. The most commonly used biomarkers of PAH exposure are urinary monohydroxy-PAH (OH-PAH). Urinary OH-PAH 
has been found to correlate well with levels of exposure to PAHs in the general populations.[24] Additional effects of human occupational exposures can be detected using urinary PAH biomarkers, independent of smoking.[25]

\section{PAHs and systemic inflammation, diabetes mellitus and CVD}

Considering the large number of deaths attributable to ambient air pollution, PAHs may be a significant contributor to the high prevalence and mortality rate of diabetes mellitus and cardiovascular disease. Several animal studies have reported positive associations between PAHs and inflammation [26, 27], oxidative stress [28, 29] and the development and progression of atherosclerosis.[30, 31] Exposure to PAHs triggers the Aryl hydrocarbon (AhR) -Xenobiotic response element (XRE) signaling pathway and causes expression of proinflammatory genes. It leads to inflammation that underlies development of atherosclerosis. Activation of the AhR-XRE pathway also induces expression of cytochrome P450 (CYP) and subsequently generates reactive oxygen species (ROS) and DNA adducts which contributes to endothelial injury and consequent atherosclerosis. Several studies have also suggested that PAHs may cause disruption of the endocrine system.[32]

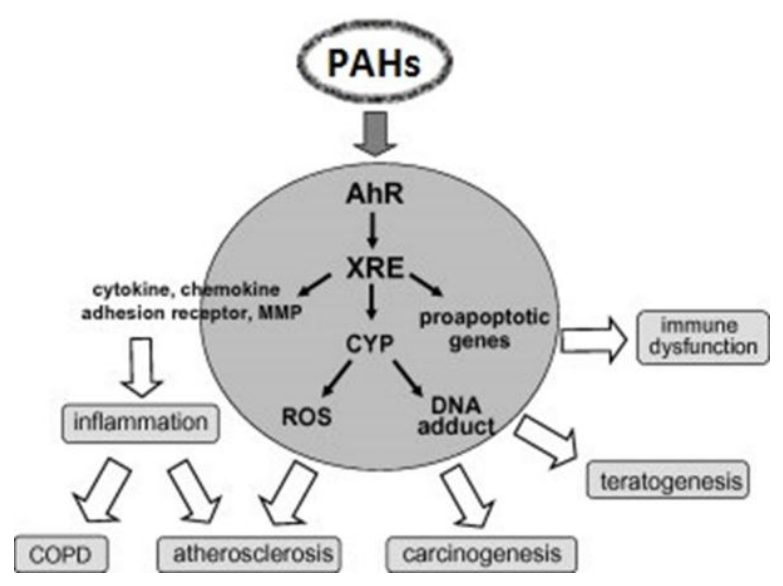

Figure 1: Masanori Kitamura and Ayumi Kasai, 2007 
In addition, several occupational studies have reported positive associations between PAHs and cardiovascular morbidity [33, 34] and mortality.[35, 36] Two general populationbased studies have investigated the association between PAHs exposure and serum markers of CVD using the National Health and Nutrition Examination Survey (NHANES), and the results were inconsistent.[37, 38] Data on PAHs were withdrawn (and re-released) due to inconsistencies in the laboratory methods used on NHANES 2001-2002 and 2003-2004 data cycles. In addition, neither adjustment for active smoking and SHS exposure in addition to confounders known to interact with the outcomes nor stratifying by major characteristics of the study population was performed in those studies. The re-release of the data provides an important opportunity for updated analysis.

The overall aim of this study was to investigate the independent association between urinary levels of PAH biomarkers, including 1-hydroxynapthol, 2- hydroxynapthol, 2hydroxyfluorene, 3-hrdroxyfluorene, 1-hydroxyphenanthrene, 2- hydroxyphenanthrene, 3hydroxyphenanthrene and 1-hydroxypyrene, and markers of systemic inflammation, diabetes mellitus and CVD, among adult participants of NHANES, a large, multiethnic, population-based, study representative of the general population of the United States adults (US). Since exposure to active smoking and SHS are major sources of PAHs, we sought to determine if this relationship was independent of serum cotinine, self-reported smoking status, in addition to the other potential confounders.

Due to the multiple sources of PAHs in the environment, exposure to a single PAH compound is implausible. Metabolism, and consequently health effects of exposure to multiple PAHs were found to be different from that of exposure to an individual PAH compound.[39] 
Enzyme competition was evident in the metabolism of PAH mixtures, changing significantly the metabolism patterns from that of individual PAHs.[39] Therefore in the current study, and similar to analytical strategies employed by previous authors [40], we created a summed variable as a measure of cumulative exposure to multiple low molecular weight PAHs simultaneously.

\section{The specific aims of the current study were as follows:}

1. To examine the independent association of urinary PAH biomarkers and serum markers of systematic inflammation such as high sensitivity C-reactive protein and white blood cell count.

2. To examine the independent association of urinary PAH biomarkers and diabetes mellitus.

3. To examine the independent association of urinary PAH biomarkers and self-reported cardiovascular disease. 


\title{
CHAPTER 1
}

\section{The Association of Urinary Levels of Polycyclic Aromatic Hydrocarbon Biomarkers and \\ Serum Inflammatory Makers.}

\begin{abstract}
Polycyclic aromatic hydrocarbons (PAHs) are potent atmospheric pollutants, occurring from both anthropogenic and natural sources. Several animal studies have reported a positive association of PAHs with inflammation. However, it is not clear if lower background exposure to PAHs in the general population is associated with systemic inflammation in humans. We examined participants from the National Health and Nutrition Examination Survey 2001-2002, 2003-2004, and 2005-2006. Our exposures were eight urinary monohydroxy polycyclic aromatic hydrocarbons $(\mathrm{OH}-\mathrm{PAH})$ and our outcomes were serum $\mathrm{C}$-reactive protein $(\mathrm{CRP})(<10 \mathrm{mg} / \mathrm{L})$ and total white blood cell (WBC) count (4000-12,000 cells/ $\mu \mathrm{L})$. Compared to those with summed biomarkers of low-molecular weight (LMW) PAHs in the lowest quartile, the multivariable odds ratios (95\% confidence interval) of serum CRP $\geq 3 \mathrm{mg} / \mathrm{L}$ and high total WBC count (defined as at or above the 95 percentile of total WBC count distribution) among those in the highest quartile were $1.77(1.13,2.76)$ and $1.34(1.12,1.60)$ respectively. Levels of 1 hydroxypyrene, the biomarker of the higher molecular weight PAH pyrene, was positively associated with total WBC count, and to lesser extent with serum CRP. In subsequent analyses, the positive association between LMW PAHs and serum CRP and total WBC count was found to be present within the stratified subgroups. In conclusion, urinary levels of PAH biomarkers were
\end{abstract}


found to be positively associated with serum CRP and total WBC count independent of smoking and other potential confounders. 
Systemic inflammation is considered a key risk factor for atherosclerosis and subsequent development of cardiovascular disease (CVD).[5] Several studies have reported a positive association between baseline elevations of C-reactive protein (CRP), a serum inflammatory maker, and future risk of CVD.[41, 42] Clinical and public health groups have recommended serum CRP levels to be used as a CVD risk stratifying tool.[43] In addition, elevations in total white blood cells (WBC) count within the normal range (4000-12,000 cells/uL) were found to be independently associated with increased risk of CVD and have been proposed as an alternate serum inflammatory marker.[44]

Polycyclic aromatic hydrocarbons (PAHs) are potent atmospheric pollutants composed of fused aromatic rings.[16, 21, 45] PAHs may occur in oil, coal, and tar deposits, and are produced as byproducts of smoking, and indoor and outdoor fuel burning.[18, 46] PAHs can be also found in contaminated water, and in food as a result of food processing, preparation, and cooking. [47, 48] Further, exposure to PAHs is markedly increased by cigarette smoking. Several in-vitro and animal studies have reported a positive association between exposure to PAHs and systemic inflammation. [28, 49-51] However, it is not clear if the lower background exposure to PAHs is associated with inflammatory effects in humans in the general population.

In this context, we examined the association of eight urinary biomarkers of PAHs, the monohydroxy-PAHs (OH-PAH), with serum CRP and total WBC count in a nationally representative sample of United States (US) adults. Since exposure to active smoking and second hand smoke are major sources of PAHs, we sought to determine if this relationship was independent of serum cotinine, self-reported smoking status, and other potential confounders. 
Due to the multiple sources of PAHs in the environment, exposure to a single PAH compound is implausible. Metabolism, and consequently health effects of exposure to multiple PAHs were found to be different from that of exposure to an individual PAH compound.[39] Enzyme competition was evident in the metabolism of PAH mixtures, changing significantly the metabolism patterns from that of individual PAHs.[39] Therefore in the current study, and similar to analytical strategies employed by previous authors [40], we created a summed variable as a measure of cumulative exposure to multiple low molecular weight PAHs simultaneously.

\section{METHODS}

\section{Study population}

The present study is based on merged data from the 2001-2002, 2003-2004 and 20052006 National Health and Nutrition Examination Survey (NHANES). Detailed description of NHANES study design and methods are available elsewhere.[52] NHANES included a stratified multistage probability sample representative of the civilian non-institutionalized US population. Selection was based on counties, blocks, households and individuals within households, and included oversampling of non-Hispanic Blacks and Mexican Americans in order to provide stable estimates of these groups. Out of 31,509 participants in NHANES 2001-2006, there were 11, 512 who were 20-65 years of age. Urinary PAH biomarkers were only measured in a subsample of individuals. The subsample is nationally representative, but with a smaller analytic sample size. 
We excluded participants with missing information on serum CRP or with CRP levels $>10 \mathrm{mg} / \mathrm{L}$, indicating potential underlying non-cardiovascular causes of inflammation.[53] We further excluded participants with missing information on serum cotinine level, or other covariates included in the final CRP model. Similarly, to minimize the confounding effect of infection, only subjects with a WBC count within the normal range (4000-12,000 cells/uL) were included in the final WBC analysis. We also excluded participants with missing information on total WBC count, or other covariates included in the final model.

\section{Main outcome of interest: Serum inflammatory markers}

\section{High sensitivity serum $C$ - reactive protein.}

Serum CRP was measured using latex-enhanced nephelometry. Details of the laboratory collection, processing, and analysis are available in the laboratory procedures manual.[54] High CRP level was defined as $\geq 3 \mathrm{mg} / \mathrm{dL}$, consistent with American Heart Association/Centers for Disease Control \& Prevention (AHA/CDC) guidelines for identifying subjects with high risk of CVD.[7]

\section{Total white blood cell count within normal values.}

The methods used to derive WBC count are based on the Beckman Coulter method of counting. High WBC count was defined as values at or above the $95^{\text {th }}$ percentile of the study population distribution, consistent with previous studies examining the association between total WBC count within normal ranges and CVD risk.[10, 55]

\section{Main exposure: Urinary levels of monohydroxy-PAH}


Urine specimens collected during the clinical exam portion of the survey were processed, stored, and shipped to the Division of Environmental Health Laboratory Sciences, National Center for Environmental Health, Centers for Disease Control and Prevention for analysis. The specific analytes measured in this study were monohydroxy-PAH (OH-PAH). By evaluating these analytes in urine, a measurement of the body burden from PAH exposure is obtained.[24] The procedure involves enzymatic hydrolysis of urine, extraction, derivatization and analysis using capillary gas chromatography combined with high resolution mass spectrometry (GCHRMS). Detailed specimen collection and processing instructions are discussed in the NHANES Laboratory/Medical Technologists Procedures Manual (LPM).

Seven LMW PAH urinary biomarkers, Naphthalene biomarkers; 1- hydroxynaphthalene, 2- hydroxynaphthalene, Fluorene biomarkers; 2-hydroxyfluorene, 3-hydroxyfluorene, Phenanthrene biomarkers; 1-hydroxyphenanthrene, 2- hydroxyphenanthrene, 3hydroxyphenanthrene and 1-hydroxypyrene, the biomarker of the higher molecular weight PAH pyrene, were consistently available in NHANES 2001-2006. All analytes were measured in the

same unit; ng/L. Urinary OH-PAH were corrected for urinary creatinine concentration, a urinary marker of kidney function to adjust for urinary dilution.[56] Urinary levels of OH-PAH (ng/L) were divided by urinary creatinine level $(\mathrm{mg} / \mathrm{dL})$ multiplied by $0.01 ;[(\mathrm{ng} / \mathrm{L}) \div(\mathrm{mg} / \mathrm{dL} * 0.01)]$ and expressed as nanogram per gram of creatinine (ng/g creatinine).

\section{Exposure measurements}

Information on age, gender, race/ethnicity, alcohol intake, income, diabetes and cigarette smoking were obtained from a standardized questionnaire during a home interview. Alcohol 
consumption was categorized into none and alcohol drinker. Income-poverty ratio (Income/poverty guideline) was used as a measure of the socioeconomic status. The Department of Health and Human Services' poverty guidelines were used as the poverty measure to calculate this ratio. Smoking status was categorized into never smokers (smoked $<100$ cigarettes during their lifetime), former smokers (smoked $\geqslant 100$ cigarettes during their lifetime and currently not smoking), current smokers (smoked $\geqslant 100$ cigarettes during their lifetime and currently smoking). Information on anthropometric, physical and laboratory components were obtained during the medical examination center examination. Body mass index (BMI) was calculated as weight in kilograms divided by height in meters squared. Seated blood pressure was measured using a mercury sphygmomanometer according to the American Heart Association and recommendations.[57] Up to 3 measurements were averaged for blood pressure.

\section{Statistical analysis}

Urinary levels of OH-PAH were analyzed both as continuous as well as categorical variables. For analysis as continuous variables, urinary $\mathrm{OH}-\mathrm{PAH}$ levels were log-transformed as a result of their skewed distribution. Weighted Pearson correlation coefficients between individual $\mathrm{OH}-\mathrm{PAH}$ were calculated to evaluate the correlations between pairwise combinations of all eight urinary metabolites. We created an additive LMW PAH biomarkers variable by summing urinary levels of metabolites of the low molecular weight PAHs (naphthalene, fluorene and phenanthrene).

We ran linear regression models to calculate the multivariable change and $95 \%$ confidence interval (CI) in serum CRP and total WBC count with increasing individual and 
additive urinary $\mathrm{OH}-\mathrm{PAH}$ levels. In addition, we ran logistic regression models to calculate the multivariable odds ratio (OR) and 95\% CI of high serum CRP ( $\geq 3 \mathrm{mg} / \mathrm{L})$ and total WBC count at or above the 95th percentile of the total WBC count distribution, for each higher urinary $\mathrm{OH}$ PAH quartile by using the lowest quartile as the referent. Variables were included in the model if they satisfied two conditions; first, a plausible association with the main exposure/outcome, and second, a $>10$ percent change of odds ratio after adjusting for the potential. Accordingly, final models were adjusted for age (years), sex (men, women), ethnicity (non-Hispanic White, nonHispanic Black, all others), poverty-income ratio (\%), alcohol drinking (yes/no), diabetes (absent/present), BMI (normal, overweight, obese), total cholesterol (mg/dL), serum cotinine (ng/mL) and systolic blood pressure $(\mathrm{mm} \mathrm{Hg})$.

To further ensure that the association is homogenous for subgroups, we performed subgroup analyses by gender, race/ethnicity, BMI and smoking categories. Sample weights that account for the unequal probabilities of selection, oversampling, and nonresponse in the NHANES survey were applied for all analyses. Analyses were conducted using SAS (version 9.3, SAS Institute, Cary, NC) software. Standard errors were estimated using the Taylor series linearization method.

\section{RESULTS}

Table 1 presents the baseline characteristics of the study population with CRP levels $<10$ $\mathrm{mg} / \mathrm{L}$. The study population was primarily non-Hispanic White (72.9\%). Approximately one-half $(50.5 \%)$ were never smokers, and the remainder were former smokers $(21.7 \%)$ and current 
smokers (27.8\%). The arithmetic mean of serum cotinine level was $74.2 \mathrm{ng} / \mathrm{mL}$. Tables 2 and 3 present the weighted percentiles and means of individual and additive LMW urinary OH-PAH metabolites used in the final CRP and total WBC count analyses, respectively.

Table 4 present the results of the analyses examining the correlations between pairwise combinations of all eight urinary metabolites in addition to serum cotinine. The results indicate that significant correlations exist between all $\mathrm{OH}-\mathrm{PAH}$ pairwise combinations, with Pearson correlation coefficients that ranged from 0.41 to 0.93 .

Tables 5 and 6 present the results of the linear regression analyses measuring the association between urinary OH-PAH levels, serum CRP and total WBC count respectively. Urinary levels of OH-PAH were positively associated with serum CRP levels independent of potential confounders. All the associations were statistically significant except for 1hydroxynaphthalene, 3-hydroxyphenanthrene and 1-hydroxypyrene. Similarly urinary levels of OH-PAH were positively associated with total WBC count independent of potential confounders.

Table 7 presents the odds ratio of the association between urinary levels of $\mathrm{OH}-\mathrm{PAH}$ and high CRP, defined as CRP levels $\geq 3 \mathrm{mg} / \mathrm{L}$. Overall, urinary levels of OH-PAH were positively associated with high CRP in the multivariable adjusted models. Using urinary OH-PAH levels as continuous variables, the observed associations were still positive. Although positive, the associations between urinary 3-hydroxyphenanthrene and serum CRP were not statistically significant.

Table 8 presents the odds ratios of the association between urinary levels of $\mathrm{OH}-\mathrm{PAH}$ and high WBC count (defined as at or above the $95^{\text {th }}$ percentile of the total WBC count distribution). 
Similar to the results for CRP, urinary levels of OH-PAH were positively associated with high total WBC count in the multivariable adjusted models. Using urinary $\mathrm{OH}-\mathrm{PAH}$ levels as continuous variables, the observed associations were still positive. Although positive, the associations between urinary 1-hydroxyphenanthrene and 3-hydroxyphenanthrene and total WBC were not statistically significant.

Table 9 investigates several types of potential confounding. We performed subgroup analyses by gender, race/ethnicity, BMI and smoking categories. Overall, consistent with the findings for the whole cohort, we found that higher urinary levels of LMW PAHs biomarkers were positively associated with high $\mathrm{CRP}$ and high total WBC count within these stratified subgroups. Compared to females, urinary biomarkers of LMW PAH showed stronger associations with serum CRP and high total WBC count among males. Compared to nonsmokers, urinary biomarkers levels of LMW PAH showed a stronger association with high total WBC count among current smokers. Some of the odds ratios failed to reach the conventional levels of statistical significance. P-interaction values for cross-product terms between urinary $\mathrm{OH}-\mathrm{PAH}$ levels and stratifying variables were all $>0.15$ except for gender in the CRP analysis $(P=0.001)$ and in the WBC analysis $(P=0.003)$.

\section{DISCUSSION}

In a multiethnic sample of US adults, we found that higher levels of urinary PAH biomarkers were positively associated with high serum CRP levels and total WBC count, independent of surveyed smoking status, serum cotinine and other potential confounders. The 
association was stronger for the low molecular weight PAH biomarkers, compared to 1hydroxypyrene, a urinary metabolite of the higher molecular weight PAH pyrene.

Researchers have examined PAHs directly in the blood and tissues of experimental animals, and in humans. The most commonly used biomarkers of PAH exposure are urinary OH-PAH. Urinary OH-PAH has been found to correlate well with levels of exposure to PAHs in the general population.[24] In addition, there is an evidence that the additional effect of human occupational exposures can be detected, independent of cigarette smoking.[25]

Humans are usually exposed to PAHs occurring in either a gas or particulate phase. PAHs with the lower molecular weight (Naphthalene, Fluorene, and Phenanthrene) are more abundant in the gas phase and are absorbed mainly through inhalation.[58] In contrast, PAHs with higher molecular weight (Pyrene) have higher vapor pressure and are found in a particulate form.[58] They can be absorbed through ingestion, skin contact and inhalation.[16, 22]

The mechanisms underlying the positive association of urinary PAH biomarkers with serum CRP and total WBC count remain unknown. Several in-vitro and animal studies have reported a positive association between exposure to PAHs and systemic inflammation. [28, 4951] Upon exposure to PAHs, detoxification occurs, leading to the formation of highly reactive intermediates that can interact with the DNA, forming PAH-DNA plaques in animal arteries. [28, 30] Several studies have suggested that PAHs might exert this atherogenic effect via stimulation of an inflammatory process involving an increased influx of proinflammatory cells into these plaques.[49]

Stratifying by gender, the positive association between urinary LMW PAH biomarkers and serum CRP $\geq 3 \mathrm{mg} / \mathrm{L}$ and total $\mathrm{WBC}$ at or above the $95^{\text {th }}$ percentile was found to be stronger 
in males when compared to females, suggesting possible gender differences. It is possible that hormonal differences in the way in which men and women metabolize PAHs may explain this observation. PAHs are known to be metabolized by cytochrome P450.[59] It has been shown that women have increased lung expression of CYP enzymes compared with men which is related to estrogen.[60, 61] Consequently, accelerated breakdown of PAHs in the lungs may potentiate the pulmonary response to PAHs in females while reducing the circulating PAH concentrations.[62] Only two human studies have investigated the association between PAH exposure and serum inflammatory markers, and the results were inconsistent. In a study of 999 participants using NHANES 2003-2004, higher exposure to PAHs was associated with elevated levels of serum CRP.[37] However, in a study of participants in NHANES 2001-2004, urinary levels of $\mathrm{OH}-\mathrm{PAH}$ were not associated with other serum inflammatory markers such as total WBC count.[63] The differences in the results may be partly due to the fact that some NHANES data on PAHs were withdrawn (and re-released) due to inconsistencies in the laboratory methods used in 2001-2002 and 2003-2004 data cycles. The re-release of the data provides an important opportunity for updated analysis. In addition, previous studies included participants with CRP levels of higher than 10 and total WBC count higher than 12,000 cells/ $\mu \mathrm{L}$, where infection or autoimmune diseases might have confounding effects. Also adjustment for major confounders known to interact with inflammatory markers such as alcohol drinking, total cholesterol and socioeconomic status, and stratifying by major characteristics of the study population were not performed in these previous studies.

The current study used merged data from NHANES 2001-2002, 2003-2004 and 20052006. The strengths of the study include the relatively large multiethnic sample of the US adults, 
the high quality of NHANES data due to standardized data collection and the ability to adjust for potential confounders and to stratify by major sociodemographic characteristics. In addition, we investigated the association of PAH exposure with serum CRP and total WBC count, independent of the health effects of smoking by adjusting for serum cotinine, an objective measure of cigarette smoke exposure in addition to stratifying by self-reported smoking status. Cotinine is the principal metabolite of nicotine. [64] Serum cotinine is considered a more precise measure of exposure to cigarette smoking when compared to self-reported smoking status, [65, 66] and is considered an accurate biomarker of second-hand smoke exposure. [67]

The study has limitations as well. The cross-sectional nature of NHANES does not allow us to draw temporal or causal inferences regarding the relationship between PAHs and serum inflammatory markers. Urinary OH-PAH measurements reflect recent exposure to PAHs and do not reflect differences between the current exposure sources and the past exposure sources for each subject. Due to its short half-life, serum cotinine also reflects recent exposure to tobacco smoke. However, these biases are likely to be non-differential biases, which would minimize any associations observed.

In conclusion, lower background exposure to PAHs was found to be positively associated with serum markers of systemic inflammation, i.e. serum CRP and total WBC count independent of potential confounders. Active smoking appears to play a significant role in the association of urinary $\mathrm{OH}-\mathrm{PAH}$ and total WBC count, yet the association persists after adjustment for serum cotinine and self-reported smoking status. There is a need to replicate these findings in future prospective studies with adequate sample size. 
Table 1. Baseline characteristics of the study population (20-65 years of age) with measured urinary levels of $\mathrm{OH}-\mathrm{PAH}$ and $\mathrm{CRP} \leq 10 \mathrm{mg} / \mathrm{L}$

\begin{tabular}{lc}
\hline Characteristics & $\begin{array}{c}\text { Mean values (Std error of mean) or } \\
\text { Sample size (weighted percentages) }\end{array}$ \\
\hline Female $(\%)$ & $1136(47.7)$ \\
Age (years) & $40.9(0.34)$ \\
Race/Ethnicity $(\%)$ & $1200(72.9)$ \\
Non-Hispanic Whites & $463(10.0)$ \\
Non-Hispanic Blacks & $691(17.1)$ \\
Others & \\
Education categories $(\%)$ & $547(14.2 \%)$ \\
Less than high school & $541(24.2)$ \\
High school & $1266(61.6)$ \\
Above high school & $392(12.1)$ \\
Below poverty level $(\%)$ & \\
Smoking categories $(\%)$ & $1229(50.5)$ \\
Never & $501(21.7)$ \\
Former smokers & $624(27.8)$ \\
Current smokers & \\
Alcohol drinking $(\%)$ & $1731(77.2)$ \\
Yes & \\
Body mass index $(\%)$ & $784(36.5)$ \\
Normal weight $\left(<25.0 \mathrm{~kg} / \mathrm{m}^{2}\right)$ & $827(32.9)$ \\
Overweight $\left(25.0-29.9 \mathrm{~kg} / \mathrm{m}^{2}\right)$ & $743(30.6)$ \\
Obese $\left(\geq 30.0 \mathrm{~kg} / \mathrm{m}^{2}\right)$ & $74.2(4.25)$ \\
Serum cotinine $(\mathrm{ng} / \mathrm{mL})$ & $201.6(1.43)$ \\
Total cholesterol $(\mathrm{mg} / \mathrm{dL})$ & $119.1(0.47)$ \\
Systolic blood pressure $(\mathrm{mmHg})$ &
\end{tabular}


Table 2: Weighted percentiles of OH-PAH (ng/g creatinine) among participants included in the final analysis with serum CRP levels $\leq 10 \mathrm{mg} / \mathrm{L}$

\begin{tabular}{|c|c|c|c|c|c|c|c|c|c|}
\hline \multirow[t]{2}{*}{ Chemicals } & & & & \multicolumn{6}{|c|}{ Selected percentiles } \\
\hline & $\begin{array}{c}\text { Sample } \\
\text { size }\end{array}$ & Mean & Minimum & $10^{\text {th }}$ & $25^{\text {th }}$ & $50^{\text {th }}$ & $75^{\text {th }}$ & $90^{\text {th }}$ & Maximum \\
\hline 1- Hydroxynaphthalene & 2480 & 44914 & 50.8 & 502.6 & 843.7 & 2009.3 & 7116.1 & 17899 & 39226536 \\
\hline 2-Hydroxynaphthalene & 2488 & 6235.5 & 93.5 & 795.9 & 1341.8 & 2820.7 & 7947.9 & 16009 & 404573 \\
\hline 2-Hydroxyfluorene & 2465 & 654.4 & 2.2 & 102.4 & 143.3 & 247.8 & 751.2 & 1756.9 & 29914 \\
\hline 3-Hydroxyfluorene & 2450 & 339.0 & 1.2 & 34.5 & 52.5 & 97.6 & 407.4 & 1014.8 & 19084 \\
\hline 1-Hydroxyphenanthrene & 2469 & 215.31 & 0.7 & 60.8 & 89.3 & 139.7 & 232.7 & 371.3 & 8341.9 \\
\hline 2-Hydroxyphenanthrene & 2448 & 96.6 & 0.5 & 22.3 & 35.1 & 56.9 & 99.9 & 177.1 & 5069.4 \\
\hline 3-Hydroxyphenanthrene & 2444 & 191.5 & 0.7 & 37.7 & 55.4 & 91.8 & 176.2 & 342.0 & 20248 \\
\hline 1-Hydroxypyrene & 2461 & 136.5 & 0.9 & 22.3 & 35.5 & 66.1 & 136.6 & 272.7 & 10014 \\
\hline Summed LMW PAH biomarkers & 2369 & 54230 & 620.0 & 2119.5 & 3156.6 & 6140.5 & 18777 & 38131 & 39235478 \\
\hline
\end{tabular}


Table 3: Weighted percentiles of OH-PAH (ng/g creatinine) among participants included in the final analysis with total WBC count between 4000-12,000 cells/ $\mu \mathrm{L}$

\begin{tabular}{|c|c|c|c|c|c|c|c|c|c|}
\hline \multirow[t]{2}{*}{ Chemicals and blood markers } & & & & \multicolumn{6}{|c|}{ Selected percentiles } \\
\hline & $\begin{array}{c}\text { Sample } \\
\text { size }\end{array}$ & Mean & Minimum & $10^{\text {th }}$ & $25^{\text {th }}$ & $50^{\text {th }}$ & $75^{\text {th }}$ & $90^{\text {th }}$ & Maximum \\
\hline 1-Hydroxynaphthalene & 2620 & 43340 & 50.8 & 485.6 & 825.2 & 1963.4 & 6878.0 & 17816 & 39226536 \\
\hline 2-Hydroxynaphthalene & 2628 & 6156.1 & 9.2 & 796.5 & 1357.5 & 2825.1 & 7829.2 & 15544 & 404573 \\
\hline 2-Hydroxyfluorene & 2604 & 632.8 & 2.2 & 102.8 & 143.4 & 244.4 & 735.1 & 1687.9 & 29914 \\
\hline 3-Hydroxyfluorene & 2588 & 325.5 & 1.2 & 34.3 & 52.3 & 95.7 & 377.7 & 950.2 & 19084 \\
\hline 1-Hydroxyphenanthrene & 2610 & 213.2 & 0.7 & 60.9 & 89.7 & 140.4 & 229.3 & 366.9 & 8341.9 \\
\hline 2-Hydroxyphenanthrene & 2587 & 96.3 & 0.5 & 22.7 & 35.7 & 58.3 & 101.2 & 177.3 & 5069.4 \\
\hline 3-Hydroxyphenanthrene & 2585 & 189.1 & 0.7 & 37.5 & 54.9 & 90.0 & 170.6 & 331.0 & 20248 \\
\hline 1-Hydroxypyrene & 2600 & 133.9 & 0.9 & 22.5 & 35.4 & 65.4 & 132.0 & 267.3 & 10014 \\
\hline Summed LMW PAH biomarkers & 2505 & 52480 & 508.7 & 2122.4 & 6085.4 & 18255 & 18255 & 37021 & 39235478 \\
\hline
\end{tabular}


Table 4: Weighted Pearson correlations between log-transformed urinary levels $\mathrm{OH}-\mathrm{PAH}$ (ng/g creatinine).

\begin{tabular}{|c|c|c|c|c|c|c|c|c|}
\hline Chemicals & 1-NAP* & 2-NAP* & 2-FLUO ${ }^{+}$ & 3-FLUO ${ }^{+}$ & $1-\mathrm{PHEN}^{\delta}$ & 2-PHEN ${ }^{\delta}$ & 3 -PHEN $\delta$ & $1-\mathrm{PYR}^{\mathrm{y}}$ \\
\hline 1- Hydroxynaphthalene & 1 & 0.60 & 0.61 & 0.62 & 0.43 & 0.41 & 0.48 & 0.49 \\
\hline 2- Hydroxynaphthalene & ----- & 1 & 0.71 & 0.71 & 0.43 & 0.48 & 0.53 & 0.57 \\
\hline 2-Hydroxyfluorene & ----- & ----- & 1 & 0.93 & 0.66 & 0.68 & 0.75 & 0.73 \\
\hline 3-Hydroxyfluorene & ----- & ----- & ----- & 1 & 0.60 & 0.62 & 0.73 & 0.71 \\
\hline 1-Hydroxyphenanthrene & ----- & ----- & ----- & ----- & 1 & 0.76 & 0.78 & 0.72 \\
\hline 2-Hydroxyphenanthrene & ----- & ----- & ---- & ----- & ---- & 1 & 0.76 & 0.71 \\
\hline 3-Hydroxyphenanthrene & ----- & ----- & ----- & ----- & ----- & ----- & 1 & 0.72 \\
\hline 1-Hydroxypyrene & ----- & ----- & ----- & ----- & ----- & ----- & ----- & 1 \\
\hline
\end{tabular}


Table 5: Linear regression: Association of urinary OH-PAH levels (ng/g creatinine) and serum CRP (mg/L).

\begin{tabular}{|c|c|c|c|}
\hline Chemicals & Sample size & Log OH-PAH & Log OH-PAH \\
\hline & & Age-sex adjusted change in CRP $^{+}$ & Multivariable change in CRP $*$ \\
\hline 1- Hydroxynaphthalene & 2480 & $0.04(-0.03,0.11)$ & $0.07(-0.01,0.14)$ \\
\hline 2-Hydroxynaphthalene & 2488 & $0.15(0.04,0.26)$ & $0.14(0.04,0.25)$ \\
\hline 2-Hydroxyfluorene & 2465 & $0.14(0.05,0.24)$ & $0.16(0.06,0.26)$ \\
\hline 3-Hydroxyfluorene & 2450 & $0.07(0.00,0.14)$ & $0.10(0.02,0.18)$ \\
\hline 1-Hydroxyphenanthrene & 2469 & $0.20(0.07,0.32)$ & $0.21(0.10,0.33)$ \\
\hline 2-Hydroxyphenanthrene & 2448 & $0.24(0.05,0.43)$ & $0.19(0.01,0.36)$ \\
\hline 3-Hydroxyphenanthrene & 2444 & $0.02(-0.11,0.15)$ & $0.05(-0.06,0.17)$ \\
\hline 1-Hydroxypyrene & 2461 & $0.11(0.001,0.22)$ & $0.11(-0.004,0.21)$ \\
\hline Summed LMW PAH biomarkers & 2369 & $0.13(0.02,0.23)$ & $0.15(0.04,0.25)$ \\
\hline
\end{tabular}


Table 6: Linear regression: Association of urinary OH-PAH levels (ng/g creatinine) and total WBC count (cells/uL).

\begin{tabular}{lccc}
\hline \multicolumn{1}{c}{ Chemicals } & Sample size & Log OH-PAH & Log OH-PAH \\
\hline & & Age-sex adjusted change in WBC ${ }^{+}$ & Multivariable change in WBC * $^{*}$ \\
1- Hydroxynaphthalene & 2620 & $176.1(108.8,243.4)$ & $89.7(8.9,170.5)$ \\
2-Hydroxynaphthalene & 2628 & $345.5(233.4,457.6)$ & $229.2(103.2,355.3)$ \\
& & & $310.1(190.1,430.0)$ \\
2-Hydroxyfluorene & 2604 & $396.2(310.8,481.5)$ & $235.9(135.9,335.9)$ \\
3-Hydroxyfluorene & 2588 & $305.8(237.5,347.1)$ & $132.4(19.5,245.3)$ \\
& 2610 & $206.9(110.8,503.0)$ & $213.6(117.3,309.9)$ \\
1-Hydroxyphenanthrene & 2587 & $354.7(258.1,451.3)$ & $199.8(81.8,317.7)$ \\
2-Hydroxyphenanthrene & 2585 & $308.0(204.3,411.7)$ & $144.5(53.8,235.2)$ \\
3-Hydroxyphenanthrene & & & \\
1-Hydroxypyrene & 2600 & $283.5(201.4,365.6)$ & \\
Summed LMW PAH biomarkers & 2505 & $296.4(202.3,390.44)$ & $186.0(73.0,299.0)$ \\
\hline
\end{tabular}

${ }^{+}$Adjusted for age (years) and sex (Male/ Female).

* Adjusted for age (years), sex (Male/ Female), BMI (Normal weight/ Overweight/ Obese), race (Non-Hispanic white/ Non-Hispanic black/ all others), alcohol drinking (yes/ no), poverty-income ratio, total cholesterol (mg/dL), serum cotinine (ng/mL), diabetes mellitus (absent/ present) and systolic blood pressure ( $\mathrm{mm} \mathrm{Hg}$ ). 
Table 7. Logistic regression: Association of urinary OH-PAH levels (ng/g creatinine) and CRP $\geq 3 \mathrm{mg} / \mathrm{L}$

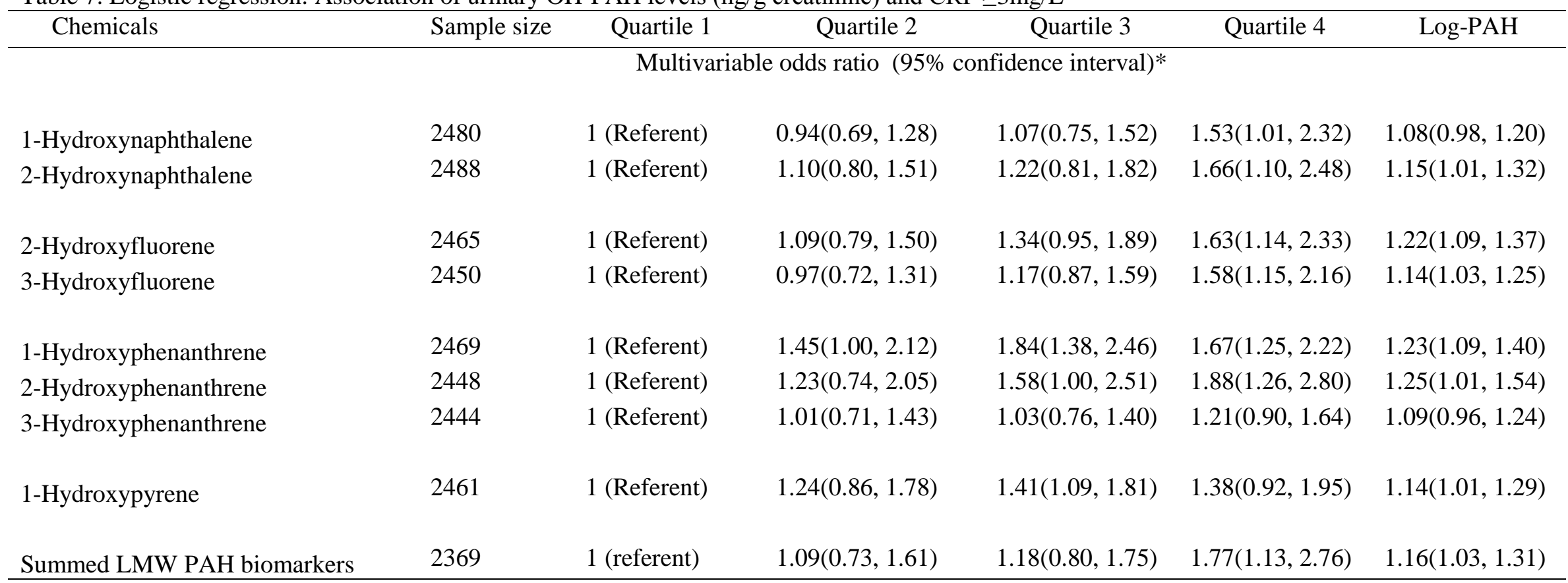

*Adjusted for age (years), sex (Male/ Female), BMI (Normal weight/ Overweight/ Obese), race (Non-Hispanic white/ Non-Hispanic black/ all others), alcohol drinking (yes/ no), poverty-income ratio, total cholesterol (mg/dL), serum cotinine (ng/mL), diabetes mellitus (absent/ present) and systolic blood pressure (mm Hg). 
Table 8. Logistic regression: Association of urinary OH-PAH levels (ng/g creatinine) and high total WBC count (cells/uL) at or above the $95^{\text {th }}$ percentile of the total WBC distribution

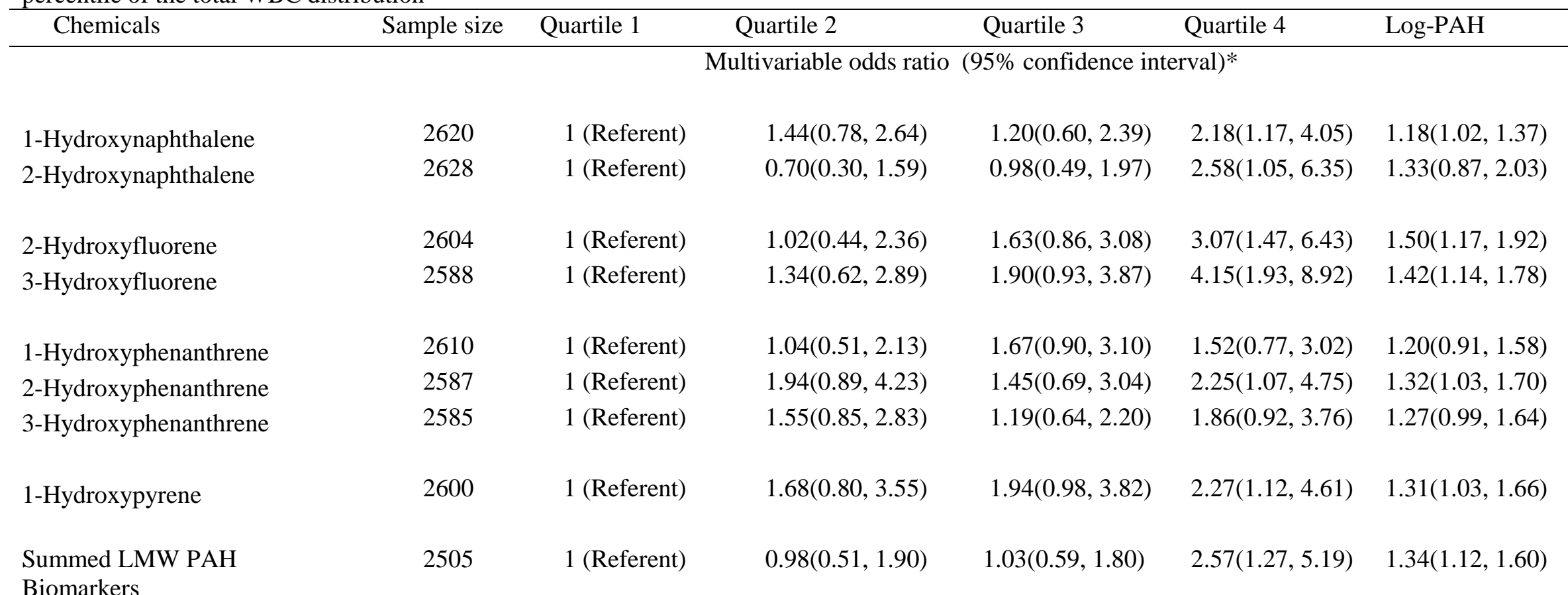

*Adjusted for age (years), sex (Male/ Female), BMI (Normal weight/ Overweight/ Obese), race (Non-Hispanic white/ Non-Hispanic black/ all others), alcohol drinking (yes/ no), poverty-income ratio, total cholesterol (mg/dL), serum cotinine ( $\mathrm{ng} / \mathrm{mL})$, diabetes mellitus (absent/ present) and systolic blood pressure ( $\mathrm{mm} \mathrm{Hg})$. 
Table 9. Logistic regression: Association of summed urinary biomarkers of LMW PAHs (ng/g creatinine) and serum inflammatory markers by sociodemographic characteristics

\begin{tabular}{|c|c|c|c|c|}
\hline Subgroups & $\begin{array}{c}\text { Multivariable odds ratio of CRP } \\
\geq 3 \mathrm{mg} / \mathrm{L}^{*}\end{array}$ & P interaction & $\begin{array}{c}\text { Multivariable odds ratio of total WBC count in } \\
\text { the } 95^{\text {th }} \text { percentile* }\end{array}$ & p-interaction \\
\hline Gender* & & 0.001 & & 0.003 \\
\hline Female & $1.08(0.93,1.25)$ & & $1.21(0.95,1.55)$ & \\
\hline Male & $1.32(1.12,1.55)$ & & $1.90(1.41,2.56)$ & \\
\hline Race-ethnicity* & & 0.29 & & 0.29 \\
\hline Non-Hispanic white & $1.16(1.00,1.34)$ & & $1.34(1.10,1.64)$ & \\
\hline All others & $1.18(0.98,1.41)$ & & $1.27(0.92,1.74)$ & \\
\hline Body mass index ${ }^{+}$ & & 0.55 & & 0.71 \\
\hline Non obese & $1.20(1.01,1.41)$ & & $1.39(1.11,1.75)$ & \\
\hline Obese & $1.08(0.91,1.27)$ & & $1.28(1.00,1.64)$ & \\
\hline Smoking* & & 0.27 & & 0.35 \\
\hline Never smokers & $1.21(0.98,1.50)$ & & $1.21(0.88,1.68)$ & \\
\hline Former smokers & $1.03(0.88,1.19)$ & & $1.15(0.83,1.59)$ & \\
\hline Current smokers & $1.10(0.81,1.49)$ & & $1.78(1.28,2.48)$ & \\
\hline
\end{tabular}

* Adjusted for age (years), sex (male, female), ethnicity (non-Hispanic white, non-Hispanic black, all others), poverty-income ratio, alcohol drinking (yes/no), diabetes (absent/present), BMI (normal, overweight, obese), total cholesterol (mg/dL), serum cotinine (ng/mL) and systolic blood pressure $(\mathrm{mm} \mathrm{Hg})$, except for stratified variables.

+ Adjusted for age (years), sex (male, female), ethnicity (non-Hispanic white, non-Hispanic black, all others), poverty-income ratio, alcohol drinking (yes/no), diabetes (absent/present), BMI $\left(\mathrm{Kg} / \mathrm{m}^{2}\right)$, total cholesterol $(\mathrm{mg} / \mathrm{dL})$, serum cotinine $(\mathrm{ng} / \mathrm{mL})$ and systolic blood $\mathrm{pressure}(\mathrm{mm} \mathrm{Hg})$, 


\title{
CHAPTER 2
}

\section{The Association of Urinary Polycyclic Aromatic Hydrocarbon Biomarkers and Diabetes Mellitus.}

\begin{abstract}
Diabetes mellitus is a major cause of morbidity and mortality in the United States. Recent evidences has suggested that common environmental exposures affecting large sections of the population may be a determinant of diabetes risk. Polycyclic aromatic hydrocarbons (PAHs) are a group of compounds produced as byproducts of incomplete combustion of organic materials. Animal studies have reported a positive association between PAHs and inflammation, and subsequent development of diabetes mellitus. Occupational studies have suggested that exposure to other aromatic hydrocarbons such as Dioxins, a group of halogenated aromatic hydrocarbons, may be associated with diabetes mellitus risk in humans. In the current study we investigate whether background exposure to PAHs is associated with increased risk of diabetes mellitus in the general population. We examined participants from the merged National Health and Nutrition Examination Survey 2001-02, 2003-2004, and 2005-2006. Our exposures of interest were eight urinary monohydroxy polycyclic aromatic hydrocarbons (OH-PAH) and our outcome was diabetes mellitus defined as a serum glucose $\geq 126 \mathrm{mg} / \mathrm{dL}$ after fasting for a minimum of 8 hours, glycosylated hemoglobin level $\geq 6.5 \%$, a self-reported physician-diagnosed diabetes, or current use of oral hypoglycemic medication or insulin. Compared to participants with summed urinary biomarkers of LMW PAHs in the lowest quartile, the multivariable odds ratio (95\% confidence interval) of diabetes mellitus among those in the highest quartile was 2.79 (1.46,
\end{abstract}


5.32). In addition, urinary levels of 2-hydroxynapthalene, 2-hyrdroxyfluorene, and 2hydroxyphenanthrene showed statistically significant associations with fasting blood glucose $(\mathrm{mg} / \mathrm{dl})$ and glycosylated hemoglobin $(\%)$, independent of potential confounders. Levels of 1hydroxypyrene, the urinary biomarker of the higher molecular weight PAH pyrene, showed a less strong association with diabetes mellitus. 
Diabetes mellitus is the seventh leading cause of death in the US.[30] Approximately 8.3\% of the American population has diabetes.[30] Diabetes is a major risk factor for cardiovascular disease, kidney failure, non-traumatic lower limb amputations, and retinopathy among adults in the US.[2, 3] Recent studies have suggested that common environmental exposures affecting large sections of the population may be a determinant of diabetes risk.[13]

Polycyclic aromatic hydrocarbons (PAHs) are a group of atmospheric pollutants composed of aromatic rings.[16, 21, 45] Major sources of PAHs include coal, oil, tar, cigarette smoking and grilled/smoked food.[47, 48, 68] PAHs are usually found in gaseous or particulate form. Low molecular weight (LMW) PAHs (naphthalene, Fluorene and Phenanthrene) are usually found in gaseous form in the ambient air where they are absorbed by inhalation. Higher molecular weight PAHs (pyrene) are usually found in particulate form where they can be ingested, inhaled or absorbed through the skin.[22, 69]

Several in-vitro and animal studies have reported a positive association between exposure to PAHs, and inflammation with subsequent development of diabetes mellitus.[70] In addition, several studies have suggested that exposure to other aromatic hydrocarbons such as Dioxins, a group of halogenated aromatic hydrocarbons is associated with diabetes mellitus in humans.[14] However, there are no studies investigating the association of lower background exposure to PAHs with diabetes mellitus in the general population independent of smoking, a major source of PAHs.

In this context, we examined the association between eight urinary monohydroxy-PAH (OH-PAH) and diabetes mellitus in a nationally representative sample of United States (US) adults after adjusting for age, gender, race/ethnicity, body mass index(BMI), serum cotinine, 
self-reported smoking status and other potential confounders. Because metabolism and health effects of exposure to PAHs mixtures were found to be different from that of exposure to individual PAHs [39], we created a summed LMW PAH biomarker variable, indicating a graduating level of exposure to multiple LMW PAHs simultaneously.

\section{METHODS}

\section{Study population}

The present study is based on merged data from the 2001-2002, 2003-2004 and 2005-2006 National Health and Nutrition Examination Survey (NHANES). NHANES surveys were designed to be nationally representative of the non-institutionalized U.S. civilian population by using a complex stratified multistage probability sample.[71] Selection was based on counties, blocks, households and individuals within households, and included oversampling of nonHispanic Blacks and Mexican Americans in order to provide stable estimates of these groups. Data were collected from interviews, questionnaire, examinations, and laboratory tests of biological samples. The data have been released for public use in two-year increments since 1999.

Out of 31,509 participants in NHANES 2001-2006, there were 3326 participants, 20-65 years of age with measured levels of urinary PAH biomarkers. We excluded participants with missing information on serum cotinine level or other variables included in the multivariable model. This resulted in 2661 participants included in the additive LMW PAH-diabetes analyses. We excluded 
those on oral hypoglycemic medications or insulin to avoid the confounding effect of antidiabetic medications, resulting in 2583 participants included in the added PAH biomarkersHbA1c analysis and 1275 participants included in the added PAH biomarkers- fasting blood glucose analyses

\section{Main outcome of interest: Diabetes mellitus}

Plasma glucose concentration was determined by a hexokinase method. One-half of the NHANES participants were sampled to attend the morning session. Those participants ages 12 and older appointed to attend the morning session were instructed to fast at least 9 hours prior to their appointment time. Glycohemoglobin measurements for NHANES 2001-2002 and 20032004 were performed by the Diabetes Diagnostic Laboratory at the University of MissouriColumbia using Primus CLC330 and Primus CLC 385 (Primus Corporation, Kansas City, MO). Glycohemoglobin measurements for NHANES 2005-2006 were performed by the Diabetes Laboratory at the University of Minnesota using Tosoh A1c 2.2 Plus Glycohemoglobin Analyzer (Tosoh Medics, Inc., San Francisco, CA). Both assays use a High Performance Liquid Chromatography (HPLC) system.

Diabetes was defined based on the guidelines of the American Diabetes Association as a serum glucose $\geq 126 \mathrm{mg} / \mathrm{dL}$ after fasting for a minimum of 8 hours, glycosylated hemoglobin level $\geq 6.5 \%$ a self-reported physician-diagnosed diabetes, or current use of oral hypoglycemic medication or insulin.[72] Main exposure: Urinary levels of monohydroxy-PAH 
Urine specimens are processed, stored, and shipped to the Division of Environmental Health Laboratory Sciences, National Center for Environmental Health, Centers for Disease Control and Prevention for analysis. The specific analytes measured in this method are monohydroxy-PAH (OH-PAH). By evaluating these analytes in urine, a measurement of the body burden from PAH exposure is obtained. The procedure involves enzymatic hydrolysis of urine, extraction, derivatization and analysis using capillary gas chromatography combined with high resolution mass spectrometry (GC-HRMS). Detailed specimen collection and processing instructions are discussed in the NHANES Laboratory/Medical Technologists Procedures Manual (LPM).

Eight urinary OH-PAH analytes, 1- hydroxynaphthalene, 2- hydroxynaphthalene, 2hydroxyfluorene, 3-hrdroxyfluorene, 1-hydroxyphenanthrene, 2- hydroxyphenanthrene, 3hydroxyphenanthrene and 1-hydroxypyrene, are consistently available in NHANES 2001-2006. All analytes were measured in the same unit, ng/L. Urinary OH-PAH were corrected for creatinine concentration, a urinary marker of kidney function to adjust for urinary dilution [56]. Urinary levels of OH-PAH $(\mathrm{ng} / \mathrm{L})$ were divided by urinary creatinine level $(\mathrm{mg} / \mathrm{dL})$ multiplied by 0.01 i.e.; $[(\mathrm{ng} / \mathrm{L}) \div(\mathrm{mg} / \mathrm{dL} * 0.01)]$, and expressed as nanogram per gram of creatinine $(\mathrm{ng} / \mathrm{g}$ creatinine).

\section{Exposure measurements}

Information on age, gender, race/ethnicity, alcohol intake, income and cigarette smoking were obtained from a standardized questionnaire during a home interview. Alcohol consumption was categorized into none and alcohol drinker. Income-poverty ratio was used as a measure of the socioeconomic status. The Department of Health and Human Services' poverty guidelines 
were used as the poverty measure to calculate this ratio. Smoking status was categorized into never smokers (smoked $<100$ cigarettes during their lifetime), former smokers (smoked $\geqslant 100$ cigarettes during their lifetime and currently not smoking), current smokers (smoked $\geqslant 100$ cigarettes during their lifetime and currently smoking). Information on anthropometric, physical and laboratory components were obtained during the medical examination center examination. Body mass index was calculated as weight in kilograms divided by height in meters squared. Seated blood pressure was measured using a mercury sphygmomanometer according to the American Heart Association and JNC7 recommendations.[57] Up to 3 measurements were averaged for blood pressure. Total cholesterol was measured enzymatically. Serum cotinine was measured by isotope dilution-high performance liquid chromatography atmospheric pressure chemical ionization tandem mass spectrometry (ID HPLC-APCI MS/MS).

\section{Statistical analysis}

Urinary levels of OH-PAH were analyzed both as continuous as well as categorical variables. For analysis as continuous variables, urinary levels of $\mathrm{OH}-\mathrm{PAH}$ were log-transformed as a result of their skewed distribution. We created a summed LMW PAH variable by adding the urinary levels of the LMW PAHs (naphthalene, fluorene and phenanthrene).

We ran linear regression models to calculate the multivariable change and $95 \%$ confidence interval (CI) in HbA1c (\%) and fasting plasma glucose (mg/dl) for increasing urinary OH-PAH levels. In addition, we ran logistic regression models to calculate the multivariable odds ratio (OR) and $95 \%$ confidence interval (CI) of diabetes mellitus, for each higher urinary $\mathrm{OH}-\mathrm{PAH}$ quartile by using the lowest quartile as the referent. Variables were included in the 
model if they satisfied two conditions: first, a plausible association with the main outcome that is not causal, and second, if the percent change of the odds ratio after adjusting for the potential confounder was over $10 \%$. Accordingly, final models were adjusted for age (years), sex (men, women), ethnicity (non-Hispanic White, non-Hispanic Black, all others), poverty-income ratio, alcohol drinking (yes/no), BMI (normal, overweight, obese), total cholesterol (mg/dL), serum cotinine (ng/mL) and systolic blood pressure ( $\mathrm{mm} \mathrm{Hg})$.

To further ensure that the association was parallel for subgroups, we performed subgroup analyses by gender, race/ethnicity, BMI and smoking categories. Sample weights that account for the unequal probabilities of selection, oversampling, and nonresponse in the NHANES survey were applied for all analyses. Analyses were conducted using SAS (version 9.3, SAS Institute, Cary, NC) software. Standard errors were estimated using the Taylor series linearization method.

\section{RESULTS}

Table 1 presents the baseline characteristics of the study population. The study population was primarily non-Hispanic White (72.2\%). Normal weight, overweight, and obese BMI categories were equally distributed. Approximately one-half (50.5\%) were never smokers, and the remainder was former smokers $(21.5 \%)$ and current smokers $(26.0 \%)$. The mean of serum cotinine level was $74.4 \mathrm{ng} / \mathrm{mL}$. Diabetes was reported in $7.8 \%$ of the population. Table 2 presents the sample sizes, weighted means and weighted percentile of urinary levels of OH-PAH used in the final analysis. 
Table 3 presents the results of the analyses examining the linear association between urinary $\mathrm{OH}-\mathrm{PAH}$, and $\mathrm{HbA1c}(\%)$. Urinary levels of $\mathrm{OH}-\mathrm{PAH}$ were positively associated with HbA1c levels in the multivariable adjusted model; however, 1- hydroxynaphthalene, 1hydroxypyrene and summed LMW PAH biomarkers were not statistically significant. Table 4 presents the results of the analyses examining the linear association between urinary OH-PAH and fasting plasma glucose (mg/dl). Urinary levels of OH-PAH were positively associated with fasting blood glucose; however, only 2-hydroxynapthalene, 2-hrdroxyfluorene, 2hydroxyphenanthrene and 1-hydroxypyene were statistically significant.

Table 5 presents the odds ratio of the association between urinary levels of $\mathrm{OH}-\mathrm{PAH}$ and diabetes mellitus. Overall, urinary levels of OH-PAH were positively associated with diabetes mellitus in the multivariable adjusted models. Using urinary OH-PAH levels as continuous variables, the observed associations were still positive. Although positive, the associations between urinary 1-hydroxyphenanthrene and 1-hydroxypyrene and diabetes were not statistically significant.

Next, to examine confounding, we performed subgroup analyses by gender, race/ethnicity, BMI and smoking categories (Table 6). Overall, higher summed levels of LMW PAH biomarkers were positively associated with diabetes within the stratified subgroups. Although positive, some of the odds ratio failed to reach the statistical significance. P-interaction values for cross-product terms between urinary OH-PAH levels and stratifying variables were all $>0.10$ except for BMI $(\mathrm{P}=0.03)$. Urinary 1-hydroxypyrene levels showed a less strong 
association with diabetes mellitus within the stratified subgroups when compared to LMW PAHs.

\section{DISCUSSION}

In a relatively large multiethnic sample of US adults, we found that higher levels of urinary biomarkers of LMW PAHs were positively associated diabetes mellitus, independent of potential confounders. In addition, urinary levels of 2-hydroxynapthalene, 2-hrdroxyfluorene, and 2-hydroxyphenanthrene showed positive associations with glycosylated hemoglobin and fasting plasma glucose in the multivariable adjusted model. In subsequent subgroup analyses, the positive association was present within subgroups of gender, race, BMI and smoking status. Urinary biomarker levels of the higher molecular weight pyrene showed a less strong association with diabetes mellitus. Since LMW PAHs are usually found in gaseous form, absorption of PAHs through inhalation from the ambient air may exert the greatest effect on the association between PAHs and diabetes when compared to the higher molecular weight PAHs.

Researchers have examined PAHs directly in the blood and tissues of experimental animals; however, these methods have not been widely used in humans due to the high costs and the limited clinical significance of testing. The most commonly used biomarkers of PAH exposure are urinary OH-PAH biomarkers and PAH-DNA adducts. Urinary OH-PAH biomarkers and, to a lesser extent, PAH-DNA adducts have been found to correlate well with low background levels of exposure to PAHs.[24]

The mechanisms underlying the positive association between urinary PAH biomarkers and diabetes remain unknown. Several in-vitro and animal studies have reported a positive 
association between exposure to PAHs and subsequent development of oxidative stress.[28] Increasing evidence suggests that oxidative stress plays a major role in the pathogenesis of diabetes mellitus.[73] Animal studies have reported a positive association between PAH exposure and inflammation.[49] In addition, higher exposure to PAHs was associated with elevated levels of C-reactive protein, a serum inflammatory marker in humans.[37] Inflammation has been found to strongly associate with insulin resistance and diabetes mellitus.[74, 75] Moreover, chronic exposure to PAHs was found to cause intestinal inflammation with subsequent increase in the risk of type 2 diabetes in animals.[70] Besides its suggested inflammatory effects, several studies suggested that PAHs may cause disruption of the endocrine system, with changes in plasma estrogen concentrations in rats upon exposure to PAHs.[32] Exposure to PAHs was suggested as a predisposing factor in the etiology of breast cancer by disrupting the expression of BRCA-1 transcription in estrogen receptor-positive tumors.[76] In the same context, women with a BRCA-1 mutation were found to have a 2-fold increase in the risk of developing diabetes.[77]

The current study has strengths including the relatively large multiethnic sample of US adults, the high quality of NHANES laboratory and data collection methods and the ability to adjust for potential confounders. In addition, this is the first study to investigate the association between PAHs and diabetes in humans adjusting for potential confounders including serum cotinine, an objective measure of cigarette smoke exposure. Serum cotinine is considered a more precise measure of exposure to cigarette smoking when compared to self-reported smoking status $[65,66]$ and is considered an accurate biomarker of SHS exposure.[67] 
The study has several limitations. The cross-sectional nature of NHANES does not allow us to draw temporal or causal inferences regarding the relationship between PAHs and diabetes mellitus. Urinary PAH measurements reflect recent exposure if there are great differences between the current exposure sources and the past exposure sources for each subject. However, these biases are likely to be non-differential biases, which would minimize any associations observed. In addition, because NHANES does not collect information to identify the type of diabetes, we cannot not distinguish type 1 and type 2 diabetes. However, we believe that the majority of diabetes subjects in our sample have type 2 diabetes mellitus.

In conclusion, urinary biomarker levels of LMW PAHs were found to be positively associated with diabetes mellitus, independent of potential confounders. Both PAH exposure and diabetes mellitus are highly prevalent in the United States. Considering the limitations of our study, further prospective studies are necessary to determine the causal relationship between PAH exposure and the risk of diabetes mellitus. 
Table 1. Baseline characteristics of the study population 20-65 years of age with measured urinary levels of OH-PAH $(n=2661)$

\begin{tabular}{|c|c|}
\hline Characteristics & $\begin{array}{l}\text { Mean values } \pm \text { (Std error of mean) or } \\
\text { Sample size (weighted percentages) }\end{array}$ \\
\hline Female $(\%)$ & $1354(49.6)$ \\
\hline Age (years) & $41.0 \pm(0.32)$ \\
\hline \multicolumn{2}{|l|}{ Race/Ethnicity (\%) } \\
\hline Non-Hispanic Whites & $1332(72.2)$ \\
\hline Non-Hispanic Blacks & $551(10.6)$ \\
\hline Others & $778(17.3)$ \\
\hline \multicolumn{2}{|l|}{ Education categories (\%) } \\
\hline Less than high school & 637 (14.4) \\
\hline High school & $619(24.7)$ \\
\hline Above high school & $1405(60.9)$ \\
\hline Below poverty level (\%) & $461(12.6)$ \\
\hline \multicolumn{2}{|l|}{ Smoking categories $(\%)$} \\
\hline Never & $1395(50.5)$ \\
\hline Former smokers & $559(21.5)$ \\
\hline Current smokers & 707 (26.0) \\
\hline \multicolumn{2}{|l|}{ Alcohol drinking $(\%)$} \\
\hline No & $742(23.7)$ \\
\hline Yes & $1919(76.3)$ \\
\hline \multicolumn{2}{|l|}{ Body mass index (\%) } \\
\hline Normal weight $\left(<25.0 \mathrm{~kg} / \mathrm{m}^{2}\right)$ & $824(34.3)$ \\
\hline Overweight $\left(25.0-29.9 \mathrm{~kg} / \mathrm{m}^{2}\right)$ & $901(31.8)$ \\
\hline Obese $\left(\geq 3 \mathrm{vSZ0} .0 \mathrm{~kg} / \mathrm{m}^{2}\right)$ & $936(33.9)$ \\
\hline Serum cotinine $(\mathrm{ng} / \mathrm{mL})$ & $74.4 \pm(3.84)$ \\
\hline Total cholesterol (mg/dL) & $201.3 \pm(1.33)$ \\
\hline Systolic blood pressure (mmHg) & $119.3 \pm(0.47)$ \\
\hline Diabetes $(\%)$ & $254(7.8)$ \\
\hline
\end{tabular}


Table 2: Weighted percentiles of OH-PAH (ng/g creatinine) among participants included in the final analysis

\begin{tabular}{|c|c|c|c|c|c|c|c|c|c|}
\hline \multirow[t]{2}{*}{ Chemicals } & & & & \multicolumn{6}{|c|}{ Selected percentiles } \\
\hline & $\begin{array}{c}\text { Sample } \\
\text { size }\end{array}$ & Mean & Minimum & $10^{\text {th }}$ & $25^{\text {th }}$ & $50^{\text {th }}$ & $75^{\text {th }}$ & $90^{\text {th }}$ & Maximum \\
\hline 1- Hydroxynaphthalene & 2785 & 41708 & 50.8 & 488.7 & 832.6 & 1996.3 & 7120.0 & 17952 & 39226536 \\
\hline 2-Hydroxynaphthalene & 2794 & 6263.3 & 9.2 & 798.4 & 1356.5 & 2885.2 & 7952.5 & 16001 & 404573 \\
\hline 2-Hydroxyfluorene & 2767 & 651.3 & 2.2 & 102.8 & 144.0 & 245.6 & 752.1 & 1740.4 & 29914 \\
\hline 3-Hydroxyfluorene & 2751 & 334.5 & 1.2 & 34.4 & 52.3 & 96.7 & 400.7 & 1004.8 & 19084 \\
\hline 1-Hydroxyphenanthrene & 2773 & 214.1 & 0.7 & 60.8 & 89.4 & 140.9 & 234.6 & 370.2 & 8341.9 \\
\hline 2-Hydroxyphenanthrene & 2747 & 96.6 & 0.5 & 22.7 & 35.7 & 58.4 & 101.7 & 178.4 & 5069.4 \\
\hline 3-Hydroxyphenanthrene & 2745 & 188.5 & 0.7 & 37.6 & 55.0 & 90.7 & 173.8 & 333.7 & 20248 \\
\hline 1-Hydroxypyrene & 2764 & 134.4 & 0.9 & 22.5 & 35.8 & 65.9 & 136.6 & 270.4 & 10014 \\
\hline Summed LMW PAH biomarkers & 2661 & 50970 & 508.8 & 2131.3 & 3180.1 & 6207.5 & 18772 & 38592 & 39235478 \\
\hline
\end{tabular}


Table 3 Linear regression: Association of urinary OH-PAH (ng/g creatinine) and HbA1c (\%)

\begin{tabular}{|c|c|c|c|}
\hline Chemicals & Sample size & $\begin{array}{l}\text { Age-sex adjusted change (95\% C.I) } \\
\text { in HbA1c+ }\end{array}$ & $\begin{array}{l}\text { Multivariable adjusted change (95\% C.I) } \\
\text { in HbA1c* }\end{array}$ \\
\hline 1-Hydroxynaphthalene & 2707 & $-0.01(-0.03,0.01)$ & $0.01(-0.01,0.03)$ \\
\hline 2-Hydroxynaphthalene & 2715 & $0.02(-0.003,0.40)$ & $0.03(0.004,0.06)$ \\
\hline 2-Hydroxyfluorene & 2688 & $0.01(-0.01,0.04)$ & $0.05(0.02,0.09)$ \\
\hline 3-Hydroxyfluorene & 2672 & $0.004(-0.01,0.03)$ & $0.04(0.01,0.07)$ \\
\hline 1-Hydroxyphenanthrene & 2694 & $0.02(-0.03,0.06)$ & $0.05(0.002,0.10)$ \\
\hline 2-Hydroxyphenanthrene & 2668 & $0.05(0.02,0.08)$ & $0.06(0.02,0.10)$ \\
\hline 3-Hydroxyphenanthrene & 2643 & $0.02(-0.01,0.05)$ & $0.05(0.01,0.09)$ \\
\hline 1-Hydroxypyrene & 2685 & $0.01(-0.02,0.04)$ & $0.03(-0.01,0.06)$ \\
\hline Summed LMW PAH biomarkers & 2583 & $0.00(-0.02,0.02)$ & $0.01(-0.01,0.04)$ \\
\hline
\end{tabular}

\footnotetext{
*Adjusted for age (years), sex (male, female), race/ethnicity (non-Hispanic White, non-Hispanic Black, all others), poverty income ratio, alcohol
} drinking (yes/no), BMI (normal, overweight, obese), total cholesterol (mg/dL), serum cotinine $(\mathrm{ng} / \mathrm{mL}$ ) and systolic blood pressure (mm $\mathrm{Hg}$ ). 
Table 4 Linear regression: Association of urinary OH-PAH (ng/g creatinine) and fasting glucose levels (mg/dL)

\begin{tabular}{|c|c|c|c|}
\hline Chemicals & Sample size & $\begin{array}{l}\text { Age-sex adjusted change ( } 95 \% \text { C.I) in } \\
\text { fasting glucose+ }\end{array}$ & $\begin{array}{l}\text { Multivariable adjusted change (95\% C.I) in } \\
\text { fasting glucose* }\end{array}$ \\
\hline 1-Hydroxynaphthalene & 1339 & $-0.08(-0.92,0.77)$ & $0.50(-0.66,1.65)$ \\
\hline 2-Hydroxynaphthalene & 1342 & $1.11(0.10,2.11)$ & $1.95(0.26,3.63)$ \\
\hline 2-Hydroxyfluorene & 1325 & $0.87(-0.45,2.19)$ & $2.50(0.28,4.72)$ \\
\hline 3-Hydroxyfluorene & 1316 & $0.29(-0.83,1.41)$ & $1.75(-0.16,3.65)$ \\
\hline 1-Hydroxyphenanthrene & 1326 & $2.14(-0.57,4.84)$ & $2.88(-0.11,5.87)$ \\
\hline 2-Hydroxyphenanthrene & 1317 & $2.49(0.50,4.47)$ & $2.82(0.53,5.11)$ \\
\hline 3-Hydroxyphenanthrene & 1314 & $0.95(-0.94,2.83)$ & $2.15(-0.29,4.59)$ \\
\hline 1-Hydroxypyrene & 1322 & $1.34(-0.36,3.04)$ & $2.15(0.01,4.30)$ \\
\hline Summed LMW PAH biomarkers & 1275 & $0.27(-0.82,1.36)$ & $1.16(-0.50,2.83)$ \\
\hline
\end{tabular}


Table 5: Logistic regression: Association of urinary OH-PAH (ng/g creatinine) and diabetes mellitus

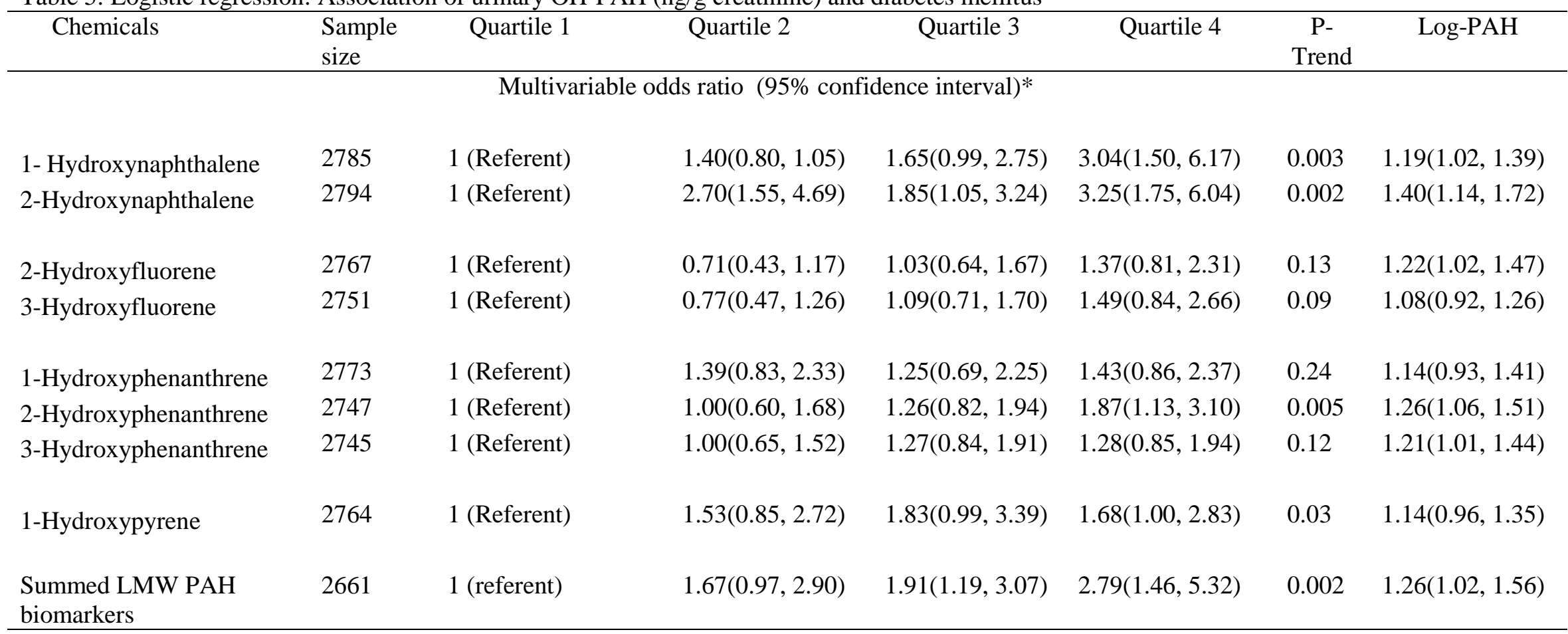

*Adjusted for age (years), sex (male, female), BMI (normal weight, overweight, obese), race (non-Hispanic White, non-Hispanic Black, all others), alcohol drinking (yes/ no), poverty-income ratio, total cholesterol $(\mathrm{mg} / \mathrm{dL})$, serum cotinine $(\mathrm{ng} / \mathrm{mL})$ and systolic blood pressure $(\mathrm{mm} \mathrm{Hg})$. 
Table 6. Logistic regression: Association of urinary OH-PAH (ng/g creatinine) and diabetes mellitus by sociodemographic characteristics

\begin{tabular}{|c|c|c|c|c|}
\hline \multirow[t]{2}{*}{ Subgroups } & \multicolumn{2}{|c|}{ Summed urinary levels LMW PAH biomarkers } & \multicolumn{2}{|l|}{ 1-hydroxypyrene } \\
\hline & $\begin{array}{c}\text { Multivariable odds ratio of diabetes } \\
\text { mellitus }\end{array}$ & p-interaction & $\begin{array}{c}\text { Multivariable odds ratio of diabetes } \\
\text { mellitus }\end{array}$ & p-interaction \\
\hline Gender* & & 0.51 & & 0.27 \\
\hline Female & $1.22(0.92,1.62)$ & & $0.97(0.73,1.27)$ & \\
\hline Male & $1.38(1.07,1.78)$ & & $1.26(1.002,1.58)$ & \\
\hline Race-ethnicity* & & 0.97 & & 0.45 \\
\hline Non-Hispanic White & $1.27(1.00,1.63)$ & & $1.13(0.91,1.40)$ & \\
\hline All others & $1.19(0.90,1.56)$ & & $1.18(0.94,1.48)$ & \\
\hline Body mass index ${ }^{+}$ & & 0.03 & & 0.19 \\
\hline Non Obese & $1.18(0.85,1.64)$ & & $1.20(0.98,1.48)$ & \\
\hline Obese & $1.26(0.96,1.66)$ & & $1.05(0.81,1.37)$ & \\
\hline Smoking* & & 0.73 & & 0.33 \\
\hline Never smokers & $1.26(1.01,1.56)$ & & $0.99(0.77,1.28)$ & \\
\hline Former smokers & $1.14(0.88,1.49)$ & & $1.32(1.00,1.75)$ & \\
\hline Current smokers & $1.63(0.96,2.79)$ & & $1.36(0.93,1.99)$ & \\
\hline
\end{tabular}




\title{
CHAPTER 3
}

\section{The Association of Urinary Polycyclic Aromatic Hydrocarbon Biomarkers and \\ Cardiovascular Disease.}

\begin{abstract}
Polycyclic aromatic hydrocarbons (PAHs) are potent atmospheric pollutants produced by incomplete combustion of organic materials. Several animal studies have reported a positive association of PAHs with oxidative stress, inflammation and subsequent development of atherosclerosis, a major underlying risk factor for cardiovascular disease (CVD). In addition, several occupational studies have reported positive associations between PAH exposure and CVD morbidity and mortality. However, it is not clear if lower background exposure to PAHs in the general population is associated with CVD. We examined participants from the merged National Health and Nutrition Examination Survey 2001-02, 2003-2004, and 2005-2006. Our exposures of interest were eight urinary monohydroxy polycyclic aromatic hydrocarbons $(\mathrm{OH}-$ PAH) and our outcomes were self-reported CVD. Urinary levels of PAH biomarkers were positively associated with CVD, however only 1-hydroxynapthalene and additive biomarkers of low molecular weight (LMW) PAHs were statistically significant. Compared to those with summed urinary biomarkers of LMW PAHs in the lowest quartile, the multivariable odds ratio (95\% confidence interval) of self-reported CVD among those in the highest quartile was 2.27(1.03, 5.02). Levels of 1-hydroxypyrene, the urinary biomarker of the higher molecular weight PAH pyrene, also showed a significant positive association with CVD. In subsequent
\end{abstract}


subgroup analyses, the positive association was found to be present in subgroups of gender, race/ethnicity, body mass index and smoking status. This study suggested that background exposure to PAHs was positively associated with self-reported CVD in the U.S. general population. Further prospective studies with adequate sample size are needed to replicate or refute our findings. 
Despite advances in prevention, diagnosis and treatment, cardiovascular disease (CVD) remains the number one cause of mortality in United States adults.[1] From a public health point of view, identifying novel risk factors for CVD is therefore important. Recent epidemiological evidence suggests that exposure to particulate matter present in ambient air is associated with increased CVD risk.[78, 79] In addition, there is increasing evidence that smaller particles and gases may pose a greater CVD risk possibly through development of atherosclerosis.[80]

Polycyclic aromatic hydrocarbons (PAHs) are potent atmospheric pollutants that occur in oil, coal, and tar deposits, and are produced as byproducts of smoking, indoors and outdoors fuel burning and food grilling.[18, 46-48] Active smoking and second-hand smoke (SHS) exposure are major sources of PAHs. PAHs are predominantly found in gaseous or particulate form. Low molecular weight (LMW) PAHs are abundant in gaseous form in the ambient air and are usually absorbed into the human body through inhalation. The higher molecular weight PAHs are usually found in particulate form and therefore, can be ingested, inhaled or absorbed through the skin.[22, 69]

Several animal studies have reported a positive association between exposure to PAHs, oxidative stress, inflammation and development of atherosclerosis[28, 30, 50, 51], a major underlying risk factor for CVD.[81-83] In addition, occupational studies have reported positive associations between exposure to PAHs and CVD morbidity and mortality.[33-36] Only one general population based study has investigated the association between PAH exposure and CVD, reporting a positive association between PAHs exposure and CVD; however adjustment for major confounders known to interact with CVD such as diabetes mellitus and cholesterol 
levels and stratification by major characteristics of the study population were not reported in this paper. [84]

In this context, using a nationally representative sample of United States (US) adults, we examined the association of eight urinary biomarkers of PAHs; specifically, monohydroxy-PAH $(\mathrm{OH}-\mathrm{PAH})$ and self-reported CVD, independent of serum cotinine, and other potential confounders. Since exposure to active smoking and SHS are major sources of PAHs, we sought to determine if this relationship was independent of serum cotinine, self-reported smoking status, and other potential confounders. Because metabolism and health effects of exposure to PAHs mixtures were found to be different from that of exposure to individual PAHs [39], we additionally created a summed LMW PAH biomarker variable, indicating a graduating level of exposure to multiple LMW PAHs simultaneously.

\section{METHODS}

\section{Study population}

The National Health and Nutrition Examination Survey (NHANES) consists of a series of surveys designed by the National Center for Health Statistics (NCHS) to continuously monitor the health status of the U.S. civilian non-institutionalized US population. The NHANES survey includes a stratified multistage probability sample. Selection is based on counties, blocks, households and individuals within households, and included oversampling of non-Hispanic Blacks and Mexican Americans in order to provide stable estimates of these groups. After all 
data are collected for a two-year survey cycle, data files are made available for analysis via the NHANES website.[85, 86]

In the current study we merged 2001-2002, 2003-3004 and 2005-2006 data cycles, where eight metabolites of PAHs have been consistently measured. Out of 31,509 participants in NHANES 2001-2006, there are 11, 512 who were 20-65 years of age. Urinary PAHs were only measured in a subsample of NHANES. We further excluded participants with missing information on serum cotinine and other covariates that were included in the final model. This resulted in 2661 participants in final added LMW PAH biomarkers model and 2764 participants in the final 1-hydroxypyrene model.

\section{Outcome: Self-reported cardiovascular disease}

The participant was considered as being a prevalent CVD case if she/he answered "yes" to any of the following questions: "Has a doctor or other health professional ever told you that you have: angina pectoris/ coronary heart disease/ heart attack/ stroke?" (These were 4 separate questions with the same wording style).

\section{Main exposure: Urinary levels of OH-PAH}

Urine specimens were processed, stored, and shipped to the Division of Environmental Health Laboratory Sciences, National Center for Environmental Health, Centers for Disease Control and Prevention for analysis. Detailed specimen collection and processing instructions are discussed in the NHANES Laboratory/Medical Technologists Procedures Manual (LPM) [87]. 
Vials are stored under appropriate frozen $\left(-20^{\circ} \mathrm{C}\right)$ conditions until they are shipped to the National Center for Environmental Health for testing.

Specific analytes measured in NHANES are monohydroxy-PAH (OH-PAH). The procedure involves enzymatic hydrolysis of urine, with extraction, derivatization and analysis using capillary gas chromatography combined with high resolution mass spectrometry (GCHRMS). This method uses isotope dilution with carbon-13 labeled internal standards. Ions from each analyte and each carbon-13 labeled internal standard are monitored, and the abundance of each ion is measured. The ratios of these ions are used as criteria for evaluating the data. By evaluating these analytes in urine, a measurement of the body burden from PAH exposure is obtained.

Seven urinary LMW PAH analytes, 1-hydroxynapthol, 2- hydroxynapthol, 2hydroxyfluorene, 3-hyrdroxyfluorene, 1-hydroxyphenanthrene, 2- hydroxyphenanthrene, 3hydroxyphenanthrene and one urinary high molecular weight analyte, 1-hydroxypyrene, were consistently available in NHANES 2001-2006. All analytes were measured in the same unit, ng/L. Urinary metabolites of PAHs were corrected for creatinine concentration, a urinary marker of kidney function.[88] Urinary levels of $\mathrm{OH}-\mathrm{PAH}(\mathrm{ng} / \mathrm{L})$ were divided by urinary creatinine level $(\mathrm{mg} / \mathrm{dL})$ multiplied by 0.01 , i.e., $[(\mathrm{ng} / \mathrm{L}) \div(\mathrm{mg} / \mathrm{dL} * 0.01)]$ and expressed as nanogram per gram of creatinine (ng/g creatinine).

\section{Exposure variables}

In NHANES, information on age, gender, race/ethnicity, alcohol intake, income, and cigarette smoking were obtained during a standardized questionnaire during a home interview. 
Information on anthropometric, physical and laboratory components were obtained during the medical examination center (MEC) examination. Body mass index was calculated as weight in kilograms divided by height in meters squared. Serum total cholesterol was measured enzymatically. Seated systolic and diastolic blood pressures were measured using a mercury sphygmomanometer according to the American Heart Association and JNC7 recommendations.[89] Serum cotinine was measured by an isotope dilution-high performance liquid chromatography atmospheric pressure chemical ionization tandem mass spectrometry (ID HPLC-APCI MS/MS).[87]

\section{Statistical analysis}

Exploratory data analysis techniques were used to assess the presence of any outliers, and the distribution of all continuous variables was examined. For analysis as continuous variables, urinary $\mathrm{OH}-\mathrm{PAH}$ levels were log-transformed as a result of their skewed distribution. We created a summed low molecular weight PAH biomarker variable by adding the urinary levels of the metabolites of low molecular weight PAHs (Naphthalene, Fluorene and Phenanthrene).

We ran logistic regression models to calculate the multivariable odds ratio ([OR] and 95\% confidence interval $[\mathrm{CI}]$ ) of self-reported CVD, for each higher urinary OH-PAH quartile by using the lowest quartile as the referent. Variables were included in the model if they satisfied two conditions: first, a plausible association with the main outcome that is not causal, and second, if the percent change of the odds ratio after adjusting for the potential confounder was over $10 \%$. Accordingly, final models were adjusted for age (years), sex (men, women), ethnicity (non-Hispanic White, non-Hispanic Black, all others), poverty-income ratio, alcohol drinking 
(yes/no), diabetes (absent/present), BMI (normal, overweight, obese), total cholesterol (mg/dL), serum cotinine $(\mathrm{ng} / \mathrm{mL})$ and systolic blood pressure $(\mathrm{mm} \mathrm{Hg})$.

To further ensure that the association is parallel for subgroups, we performed subgroup analyses by gender, race/ethnicity, BMI and smoking categories. Sample weights that account for the unequal probabilities of selection, oversampling, and nonresponse in the NHANES survey were applied for all analyses. Analyses were conducted using SAS (version 9.3, SAS Institute, Cary, NC) software. Standard errors were estimated using the Taylor series linearization method.

\section{RESULTS}

Table 1 presents baseline characteristics of the study population. About one half of the study population was female $(49.6 \%)$. The majority of the study population was non-Hispanic White $(72.2 \%)$. The arithmetic mean of serum cotinine was $74.4 \mathrm{ng} / \mathrm{mL}$. Table 2 presents the sample size, weighted means and percentiles of individual urinary $\mathrm{OH}-\mathrm{PAH}$ and added biomarkers of LMW PAHs.

Table 3 presents the results of the analyses measuring the association between urinary OH-PAH levels and self-reported CVD. Higher quartiles of 1-hydroxynapthalene and additive biomarkers of LMW PAHs showed positive association with self-reported CVD, when compared to the lowest quartiles. Using urinary levels of $\mathrm{OH}-\mathrm{PAH}$ as continuous variables, 1hydroxypyrene showed statistically significant positive association with self-reported CVD. 
Table 4 presents the association between urinary OH-PAH and self-reported CVD by major sociodemographic characteristics. The positive association between added urinary biomarkers of LMW PAHs and self-reported CVD was present among subgroups of gender, race, BMI and smoking. The positive association was stronger among the non-obese subgroup when compared to the obese group and in current smokers when compared to never and former smokers. P-interaction values for cross-product terms between added urinary biomarkers of LMW PAHs and stratifying variables were above $>0.10$ except for BMI $(P=0.002)$ and smoking $(P=0.002)$. Urinary levels of 1-hydroxypyrene, the biomarker of the higher molecular weight PAH pyrene, showed positive association with self-reported CVD within subgroups of gender, race, $\mathrm{BMI}$ and smoking. Some odds ratio failed to reach the conventional levels of statistical significance. P-interaction values for cross-product terms between urinary levels of 1hydroxypyrene and stratifying variables were above $>0.10$ except for race $(P=0.01)$.

\section{DISCUSSION}

Urinary levels of 1-hydroxynapthalene, 1-hydroxypyrene and added metabolites of LMW PAH showed positive association with self-reported CVD independent of serum cotinine and other potential confounders. The positive association was found to be stronger in the non-obese subgroup when compared to the obese subgroup and among the current smoker subgroup when compared with the never smoker and the former smoker subgroups. Our results are consistent with findings from previous occupational studies which reported positive associations between exposure to PAHs and ischemic heart diseases $[33,34]$ and cardiovascular mortality in 
occupations that include likely exposure to PAHs such as aluminum smelting, motor vehicle operators, tar distillation, and asphalt workers.[35, 36]

Polycyclic aromatic hydrocarbons are a group of chemicals formed by the incomplete combustion organic substances.[16, 21, 45] Exposure to PAHs in the environment is widespread. Human are usually exposed to mixtures of PAHs.[22] Due to the high cost of detecting parent PAH levels in humans, the most commonly used biomarkers of PAH exposure are urinary $\mathrm{OH}-$ PAH metabolites. Urinary OH-PAH biomarkers were used in the current analyses and have been found to correlate well with levels of exposure to PAHs in the general populations. [24]

Several animal studies have reported positive associations between exposure to PAHs, oxidative stress and subsequent development of atherosclerosis[28, 30, 50, 51], a major underlying risk factor for CVD.[81-83] Upon exposure to PAHs, detoxification occurs, leading to the formation of highly reactive intermediates that can interact with the DNA.[28] Accordingly, PAH exposure was found to be associated with high levels of PAH-DNA adducts in arteries in rats.[30] These effects of PAHs exposure on plaque buildup in animals were found to be dose dependent.[50] Moreover, animal studies have suggested that PAHs might also exert their atherogenic effect via stimulation of an inflammatory process involving an increased influx of proinflammatory cells into these plaques.[30] The role of inflammation as a risk factor for atherosclerosis and CVD development has become well established.[7] Several studies have shown a positive association between markers of inflammation such as total white blood cell count and serum $\mathrm{C}$ - reactive protein and CVD.[90, 91] 
In the current study, we performed subgroup analysis by gender, race/ethnicity, BMI and smoking status categories with the intent to examine confounding. In the post-hoc analyses by $\mathrm{BMI}$, the positive association between urinary $\mathrm{OH}-\mathrm{PAH}$ and self-reported CVD was found to be stronger in the non-obese subgroup. PAHs are known to have high affinity for lipid-rich tissues such as mammary and adipose tissues. [92] The suggested mechanism for the association between PAH exposure and CVD is through inflammatory PAH-DNA adducts buildup in the circulatory system. Persons with relatively higher levels of body fat are likely to exhibit relatively lower PAH activity in the circulation because of the rapid absorption of PAHs by fatty tissue. [92] Accordingly, higher levels of PAHs in the circulation may be related to higher inflammatory reaction and eventually the development of cardiovascular diseases. However, it is possible that the differences we observed are due to random variability and not true causal differences. Larger studies are needed to confirm if these observed differences in our study are consistently observed. Similarly in subgroup analysis by smoking status, current smokers showed stronger association between urinary $\mathrm{OH}-\mathrm{PAH}$ levels and CVD, when compared to never and former smokers. This stronger association may be explained by the fact that smoking is a major risk factor for cardiovascular morbidity and mortality. The population attributable risk of the smoking of tobacco products on CVD risk has been estimated at 26\%.[93]

The main strengths of our study include inclusion of a representative multiethnic sample, adequate sample size, and the ability to adjust for potential confounders. The high quality of NHANES data and the rigorous standardized methods used in the data collection add to the strengths of the study. The main limitation of our study is the cross sectional nature of NHANES which does not allow to draw temporal or causal inferences regarding the relationship between 
PAHs and self-reported CVD. In addition, our study does not have the data to estimate the sources of exposure to PAHs. Urinary PAH measurements reflect recent exposure to PAHs if there are great differences between the current exposure sources and the past exposure sources for each subject. Finally, CVD was ascertained by self-report. Accordingly, some recollection bias may exist. However, these biases are likely to be non-differential biases, which would minimize any observed association.

In summary, in a representative cross-sectional sample of the US population, we found that higher levels of urinary OH-PAH were positively associated with self-reported CVD. However, only 1-hydroxynapthalene, 1-hydroxypyrene and added biomarkers of LMW PAHs were statistically significant. The public health importance of our findings is that there is evidence, although limited, that background exposure to PAHs is positively associated with CVD in the US general population. If our findings are replicated in future larger prospective studies, the population-attributable risk of PAH exposure on CVD risk could potentially be high. 
Table 1. Baseline characteristics of the study population 20-65 years of age with measured urinary levels of OH-PAH $(n=2661)$

\begin{tabular}{lc}
\hline Characteristics & $\begin{array}{c}\text { Mean values } \pm \text { (Std error of mean) or } \\
\text { Sample size (weighted percentages) }\end{array}$ \\
\hline Female $(\%)$ & $1354(49.6)$ \\
Age (years) & $41.0 \pm(0.32)$ \\
Race/Ethnicity $(\%)$ & $1332(72.2)$ \\
Non-Hispanic Whites & $551(10.6)$ \\
Non-Hispanic Blacks & $778(17.3)$ \\
Others & \\
Education categories $(\%)$ & $637(14.4)$ \\
Less than high school & $619(24.7)$ \\
High school & $1405(60.9)$ \\
Above high school & $461(12.6)$ \\
Below poverty level $(\%)$ & \\
Smoking categories $(\%)$ & $1395(50.5)$ \\
Never & $559(21.5)$ \\
Former smokers & $707(26.0)$ \\
Current smokers & \\
Alcohol drinking $(\%)$ & $742(23.7)$ \\
No & $1919(76.3)$ \\
Yes & \\
Body mass index $(\%)$ & $924(34.3)$ \\
Normal weight $\left(<25.0 \mathrm{~kg} / \mathrm{m}^{2}\right)$ & $901(31.8)$ \\
Overweight $\left(25.0-29.9 \mathrm{~kg} / \mathrm{m}^{2}\right)$ & $119.3 \pm(0.47)$ \\
Obese $\left(\geq 30.0 \mathrm{~kg} / \mathrm{m}^{2}\right)$ & $138(4.9)$ \\
Serum cotinine $(\mathrm{ng} / \mathrm{mL})$ & \\
Total cholesterol $(\mathrm{mg} / \mathrm{dL})$ & \\
Systolic blood pressure $(\mathrm{mmHg})$ & \\
CVD $(\%)$ & \\
\hline & \\
\hline
\end{tabular}


Table 2: Weighted percentiles of OH-PAH (ng/g creatinine) among participants included in the final analysis

\begin{tabular}{|c|c|c|c|c|c|c|c|c|c|}
\hline \multirow[t]{2}{*}{ Chemicals } & & & & \multicolumn{6}{|c|}{ Selected percentiles } \\
\hline & $\begin{array}{c}\text { Sample } \\
\text { size }\end{array}$ & Mean & Minimum & $10^{\text {th }}$ & $25^{\text {th }}$ & $50^{\text {th }}$ & $75^{\text {th }}$ & $90^{\text {th }}$ & Maximum \\
\hline 1- Hydroxynaphthalene & 2785 & 41708 & 50.8 & 488.7 & 832.6 & 1996.3 & 7120.0 & 17952 & 39226536 \\
\hline 2-Hydroxynaphthalene & 2794 & 6263.3 & 9.2 & 798.4 & 1356.5 & 2885.2 & 7952.5 & 16001 & 404573 \\
\hline 2-Hydroxyfluorene & 2767 & 651.3 & 2.2 & 102.8 & 144.0 & 245.6 & 752.1 & 1740.4 & 29914 \\
\hline 3-Hydroxyfluorene & 2751 & 334.5 & 1.2 & 34.4 & 52.3 & 96.7 & 400.7 & 1004.8 & 19084 \\
\hline 1-Hydroxyphenanthrene & 2773 & 214.1 & 0.7 & 60.8 & 89.4 & 140.9 & 234.6 & 370.2 & 8341.9 \\
\hline 2-Hydroxyphenanthrene & 2747 & 96.6 & 0.5 & 22.7 & 35.7 & 58.4 & 101.7 & 178.4 & 5069.4 \\
\hline 3-Hydroxyphenanthrene & 2745 & 188.5 & 0.7 & 37.6 & 55.0 & 90.7 & 173.8 & 333.7 & 20248 \\
\hline 1-Hydroxypyrene & 2764 & 134.4 & 0.9 & 22.5 & 35.8 & 65.9 & 136.6 & 270.4 & 10014 \\
\hline Summed LMW PAH biomarkers & 2661 & 50970 & 508.8 & 2131.3 & 3180.1 & 6207.5 & 18772 & 38592 & 39235478 \\
\hline
\end{tabular}


Table 3: Logistic regression: Association of urinary levels of OH-PAH (ng/g creatinine) and self-reported cardiovascular disease

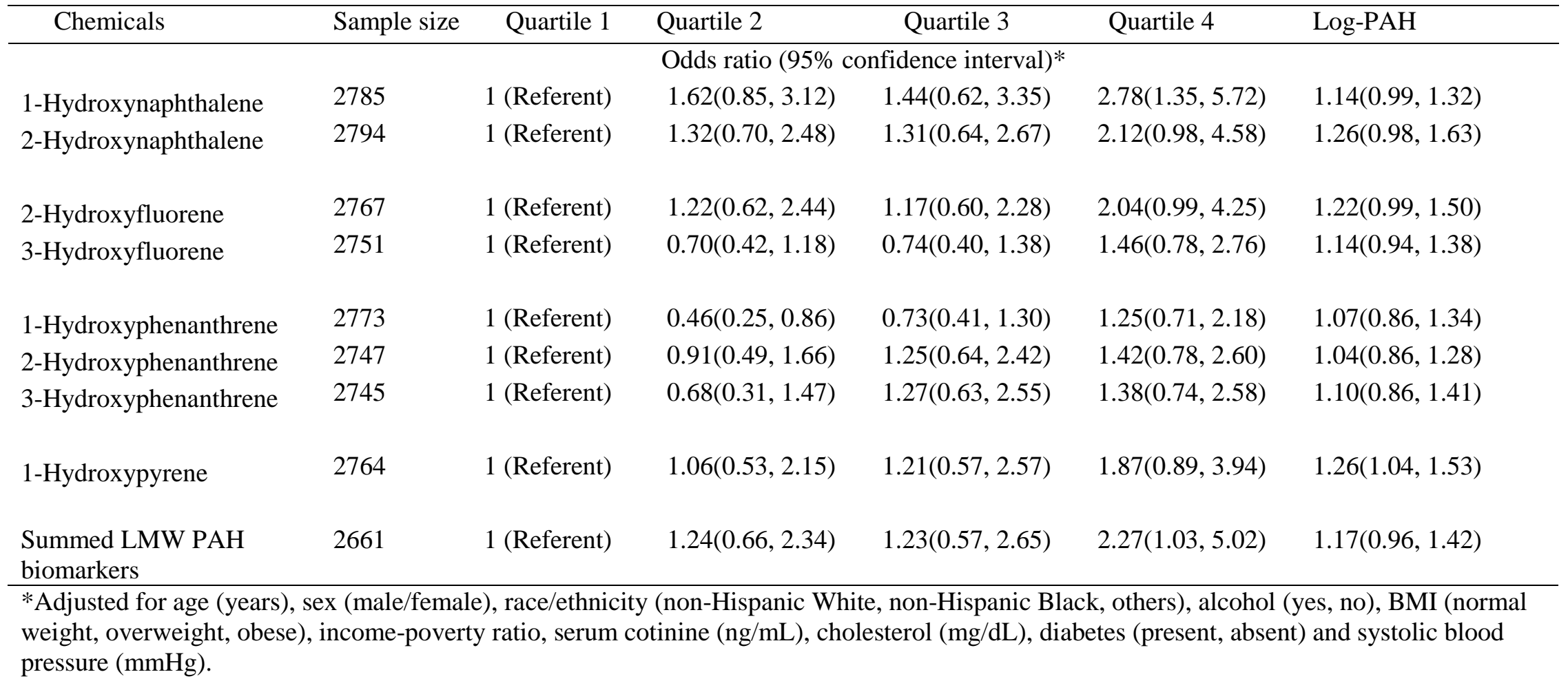


Table 4: Logistic regression: Association of urinary OH-PAH (ng/g creatinine) and self-reported cardiovascular diseases by sociodemographic characteristics

\begin{tabular}{|c|c|c|c|c|}
\hline \multirow[t]{2}{*}{ Subgroups } & \multicolumn{2}{|c|}{ Summed urinary levels LMW PAH biomarkers } & \multicolumn{2}{|c|}{ 1-hydroxypyrene } \\
\hline & Multivariable odds ratio of CVD & p-interaction & Multivariable odds ratio of CVD & p-interaction \\
\hline Gender* & & 0.64 & & 0.27 \\
\hline Male & $1.24(0.88,1.75)$ & & $1.28(1.01,1.64)$ & \\
\hline Females & $1.11(0.92,1.34)$ & & $1.25(0.89,1.73)$ & \\
\hline Race-ethnicity* & & 0.41 & & 0.01 \\
\hline Non-Hispanic whites & $1.18(0.94,1.49)$ & & $1.35(1.08,1.69)$ & \\
\hline All others & $1.14(0.81,1.60)$ & & $1.03(0.76,1.41)$ & \\
\hline Body mass index ${ }^{+}$ & & 0.002 & & 0.26 \\
\hline Non obese & $1.43(1.13,1.82)$ & & $1.39(1.05,1.82)$ & \\
\hline Obese & $1.05(0.83,1.33)$ & & $1.15(0.90,1.47)$ & \\
\hline Smoking* & & 0.002 & & 0.15 \\
\hline Never smokers & $1.21(0.93,1.56)$ & & $1.06(0.72,1.57)$ & \\
\hline Former smokers & $0.77(0.43,1.38)$ & & $1.14(0.80,1.63)$ & \\
\hline Current smokers & $2.59(1.29,5.19)$ & & $1.79(1.06,3.02)$ & \\
\hline
\end{tabular}




\section{Conclusion}

Cardiovascular disease is the number one cause death and diabetes mellitus is the seventh leading cause of death in the US adults. [72] The burden of cardiovascular disease and diabetes mellitus is continuing to grow. It has been shown that the traditional risk factors for CVD and diabetes may not explain all of the observed risk of these diseases. Environmental pollutants have been recently proposed as a novel CVD and diabetes mellitus risk factor in the general population. In the current study, urinary biomarkers of the low molecular weight polycyclic aromatic hydrocarbons (Naphthalene, Fluorene and Phenanthrene) and to lesser extent the higher molecular weight Pyrene were significantly associated with high serum C-reactive protein, high total white blood cell count, and diabetes mellitus independent of serum cotinine, self-reported smoking status and other potential confounders. The previous evidence on the association of urinary biomarker of polycyclic aromatic hydrocarbons and self-reported cardiovascular disease is limited.

These new results, consistent with previous animal and occupational studies[27], suggest that the low background exposure to polycyclic aromatic hydrocarbons may be related to low grade inflammation, evident by baseline elevations in serum CRP and total WBC count. The study also provides evidence on the associations between low background exposure to polycyclic aromatic hydrocarbons and diabetes mellitus and CVD. The mechanism underlying these associations may be through chronic low grade inflammation. Low grade inflammation has been implicated in the development of atherosclerosis and subsequent development of CVD.[7] Inflammation has been also suggested as a risk factor for insulin resistance[6] and subsequent development of diabetes mellitus.[8] 
Despite the consistency and biological plausibility, the clinical significance of our findings is still unknown. Prospective studies are necessary to confirm or refute our findings. Because exposure to polycyclic aromatic is widespread in the environment, and due to the high prevalence of diabetes and CVD, the population attributable risk due to polycyclic aromatic hydrocarbons exposure could be high. Polycyclic aromatic hydrocarbons occur from both natural as well as anthropogenic sources. Efforts to decrease the concentration of PAHs in the ambient air can be rewarding. Similar policies, such as anti-smoking bans were associated with a significant decrease in the incidence of cardiovascular disease. ${ }^{95,96}$ 


\section{REFERENCES}

1. Levy, R.I. and J. Moskowitz, Cardiovascular research: decades of progress, a decade of promise. Science, 1982. 217(4555): p. 121-9.

2. Bailes, B.K., Diabetes mellitus and its chronic complications. AORN J, 2002. 76(2): p. 266-76, 27882; quiz 283-6.

3. Bloomgarden, Z.T., Cardiovascular Disease and Diabetes. Diabetes Care, 2003. 26(1): p. 230-237.

4. Menzie, C.A., B.B. Potocki, and J. Santodonato, Exposure to carcinogenic PAHs in the environment. Environmental Science \& Technology, 1992. 26(7): p. 1278-1284.

5. Tracy, R.P., Inflammation in Cardiovascular Disease. Circulation, 1998. 97(20): p. 2000-2002.

6. Shoelson, S.E., J. Lee, and A.B. Goldfine, Inflammation and insulin resistance. The Journal of Clinical Investigation, 2006. 116(7): p. 1793-1801.

7. Pearson, T.A., et al., Markers of Inflammation and Cardiovascular Disease. Circulation, 2003. 107(3): p. 499-511.

8. Badawi, A., et al., Type 2 diabetes mellitus and inflammation: Prospects for biomarkers of risk and nutritional intervention. Diabetes Metab Syndr Obes, 2010. 3: p. 173-86.

9. Twig, G., et al., White Blood Cells Count and Incidence of Type 2 Diabetes in Young Men. Diabetes Care, 2013. 36(2): p. 276-282.

10. Twig, G., et al., White Blood Cell Count and the Risk for Coronary Artery Disease in Young Adults. PLoS One, 2012. 7(10): p. e47183.

11. Lippmann, M. and L.-C. Chen, Health effects of concentrated ambient air particulate matter (CAPs) and its components. Critical Reviews in Toxicology, 2009. 39(10): p. 865-913.

12. Poland, A. and J.C. Knutson, 2,3,7,8-tetrachlorodibenzo-p-dioxin and related halogenated aromatic hydrocarbons: examination of the mechanism of toxicity. Annu Rev Pharmacol Toxicol, 1982. 22: p. 517-54.

13. Parker, V.G., et al., Toxins and Diabetes Mellitus: An Environmental Connection? Diabetes Spectrum, 2002. 15(2): p. 109-112.

14. Wang, S.-L., et al., Increased Risk of Diabetes and Polychlorinated Biphenyls and Dioxins: A 24year follow-up study of the Yucheng cohort. Diabetes Care, 2008. 31(8): p. 1574-1579.

15. Dalton, T.P., et al., Dioxin exposure is an environmental risk factor for ischemic heart disease. Cardiovasc Toxicol, 2001. 1(4): p. 285-98.

16. Warshawsky, D., Polycyclic aromatic hydrocarbons in carcinogenesis. Environ Health Perspect, 1999. 107(4): p. 317-9.

17. Choi, H., et al., Prenatal exposure to airborne polycyclic aromatic hydrocarbons and risk of intrauterine growth restriction. Environ Health Perspect, 2008. 116(5): p. 658-65.

18. Liu, G., et al., Polycyclic aromatic hydrocarbons (PAHs) from coal combustion: emissions, analysis, and toxicology. Rev Environ Contam Toxicol, 2008. 192: p. 1-28.

19. Phillips, D.H., Polycyclic aromatic hydrocarbons in the diet. Mutat Res, 1999. 443(1-2): p. 139-47.

20. Mumtaz, M.M., et al., ATSDR evaluation of health effects of chemicals. IV. Polycyclic aromatic hydrocarbons (PAHs): understanding a complex problem. Toxicol Ind Health, 1996. 12(6): p. 742971. 
21. Angerer, J., C. Mannschreck, and J. Gundel, Biological monitoring and biochemical effect monitoring of exposure to polycyclic aromatic hydrocarbons. Int Arch Occup Environ Health, 1997. 70(6): p. 365-77.

22. Elovaara, E., et al., Significance of dermal and respiratory uptake in creosote workers: exposure to polycyclic aromatic hydrocarbons and urinary excretion of 1-hydroxypyrene. Occup Environ Med, 1995. 52(3): p. 196-203.

23. Gammon, M.D., et al., Environmental Toxins and Breast Cancer on Long Island. I. Polycyclic Aromatic Hydrocarbon DNA Adducts. Cancer Epidemiology Biomarkers \& Prevention, 2002. 11(8): p. 677-685.

24. Castano-Vinyals, G., et al., Biomarkers of exposure to polycyclic aromatic hydrocarbons from environmental air pollution. Occup Environ Med, 2004. 61(4): p. e12.

25. Ciarrocca, M., et al., Is urinary 1-hydroxypyrene a valid biomarker for exposure to air pollution in outdoor workers? A meta-analysis. J Expos Sci Environ Epidemiol, 2013/01/09.

26. Goulaouic, S., et al., Effect of polycyclic aromatic hydrocarbons and carbon black particles on pro-inflammatory cytokine secretion: impact of PAH coating onto particles. J Immunotoxicol, 2008. 5(3): p. 337-45.

27. Chuang, H.-C., et al., Vasoactive alteration and inflammation induced by polycyclic aromatic hydrocarbons and trace metals of vehicle exhaust particles. Toxicology Letters, 2012. 214(2): p. 131-136.

28. Jeng, H.A., et al., Polycyclic aromatic hydrocarbon-induced oxidative stress and lipid peroxidation in relation to immunological alteration. Occup Environ Med, 2011. 68(9): p. 653-8.

29. Liu, H., et al., An oxidative stress response to polycyclic aromatic hydrocarbon exposure is rapid and complex in Arabidopsis thaliana. Plant Science, 2009. 176(3): p. 375-382.

30. Curfs, D.M.J., et al., Polycyclic aromatic hydrocarbons induce an inflammatory atherosclerotic plaque phenotype irrespective of their DNA binding properties. The FASEB Journal, 2005.

31. Curfs, D.M., et al., Chronic exposure to the carcinogenic compound benzo[a]pyrene induces larger and phenotypically different atherosclerotic plaques in ApoE-knockout mice. Am J Pathol, 2004. 164(1): p. 101-8.

32. Archibong, A.E., et al., Alteration of pregnancy related hormones and fetal survival in F-344 rats exposed by inhalation to benzo(a)pyrene. Reprod Toxicol, 2002. 16(6): p. 801-8.

33. Tuchsen, F., et al., Occupation and ischemic heart disease in the European Community: a comparative study of occupations at potential high risk. Am J Ind Med, 1996. 30(4): p. 407-14.

34. Thériault, G.P., C.G. Tremblay, and B.G. Armstrong, Risk of ischemic heart disease among primary aluminum production workers. American Journal of Industrial Medicine, 1988. 13(6): $p$. 659-666.

35. Maclaren, W.M. and J.F. Hurley, Mortality of tar distillation workers. Scand J Work Environ Health, 1987. 13(5): p. 404-11.

36. Hansen, E.S., Mortality from cancer and ischemic heart disease in Danish chimney sweeps: a fiveyear follow-up. Am J Epidemiol, 1983. 117(2): p. 160-4.

37. Everett, C.J., et al., Association of urinary polycyclic aromatic hydrocarbons and serum C-reactive protein. Environmental Research, 2010. 110(1): p. 79-82.

38. Clark lii, J.D., et al., Exposure to polycyclic aromatic hydrocarbons and serum inflammatory markers of cardiovascular disease. Environmental Research, 2012. 117(0): p. 132-137.

39. Olatubi, A.O., Metabolism of mixtures of polycyclic aromatic hydrocarbons (PAHs) by Cunninghamella elegans. 2005, Texas A\&M University. 
40. Xia, Y., et al., Urinary metabolites of polycyclic aromatic hydrocarbons in relation to idiopathic male infertility. Human Reproduction, 2009. 24(5): p. 1067-1074.

41. Ridker, P.M., et al., Inflammation, Aspirin, and the Risk of Cardiovascular Disease in Apparently Healthy Men. New England Journal of Medicine, 1997. 336(14): p. 973-979.

42. Ridker, P.M., R.J. Glynn, and C.H. Hennekens, C-Reactive Protein Adds to the Predictive Value of Total and HDL Cholesterol in Determining Risk of First Myocardial Infarction. Circulation, 1998. 97(20): p. 2007-2011.

43. Yeboah J, M.R.L.P.T.S., COmparison of novel risk markers for improvement in cardiovascular risk assessment in intermediate-risk individuals. JAMA, 2012. 308(8): p. 788-795.

44. Kannel, W.B., K. Anderson, and P.W. Wilson, White blood cell count and cardiovascular disease. Insights from the Framingham Study. JAMA, 1992. 267(9): p. 1253-6.

45. Talaska, G., et al., Polycyclic aromatic hydrocarbons (PAHs), nitro-PAHs and related environmental compounds: biological markers of exposure and effects. Environ Health Perspect, 1996. 104 Suppl 5: p. 901-6.

46. Achten, C. and T. Hofmann, Native polycyclic aromatic hydrocarbons (PAH) in coals - A hardly recognized source of environmental contamination. Science of The Total Environment, 2009. 407(8): p. 2461-2473.

47. Ramesh, A., et al., Bioavailability and risk assessment of orally ingested polycyclic aromatic hydrocarbons. Int J Toxicol, 2004. 23(5): p. 301-33.

48. Šimko, P., Factors affecting elimination of polycyclic aromatic hydrocarbons from smoked meat foods and liquid smoke flavorings. Molecular Nutrition \& Food Research, 2005. 49(7): p. 637647.

49. Curfs, D.M., et al., Polycyclic aromatic hydrocarbons induce an inflammatory atherosclerotic plaque phenotype irrespective of their DNA binding properties. FASEB J, 2005. 19(10): p. 1290-2.

50. Penn, A., et al., Dose-dependent size increases of aortic lesions following chronic exposure to 7,12-dimethylbenz(a)anthracene. Cancer Res, 1981. 41(2): p. 588-92.

51. Albert, R.E., et al., Effect of carcinogens on chicken atherosclerosis. Cancer Res, 1977. 37(7 Pt 1): p. 2232-5.

52. Centers for Disease Control and Prevention (CDC). National Center for Health Statistics (NCHS). National Health and Nutrition Examination Survey Data. Hyattsville, M.U.S.D.o.H. and Available from: http://www.cdc.gov/nchs/nhanes/nhanes2001-2002/nhanes01 02.htm.

53. Pearson, T.A., et al., Markers of Inflammation and Cardiovascular Disease: Application to Clinical and Public Health Practice: A Statement for Healthcare Professionals From the Centers for Disease Control and Prevention and the American Heart Association. Circulation, 2003. 107(3): p. 499-511.

54. Centers for Disease Control and Prevention (CDC). National Center for Health Statistics (NCHS). National Health and Nutrition Examination Laboratory Protocol. Hyattsville, M.U.S.D.o.H.; Available from: http://www.cdc.gov/nchs/data/nhanes/nhanes 01 02/lab b generaldoc.pdf.

55. Kannel Wb, A.K.W.P.F., White blood cell count and cardiovascular disease: Insights from the framingham study. JAMA, 1992. 267(9): p. 1253-1256.

56. Barr, D.B., et al., Urinary creatinine concentrations in the U.S. population: implications for urinary biologic monitoring measurements. Environ Health Perspect, 2005. 113(2): p. 192-200.

57. Chaturvedi, S., The Seventh Report of the Joint National Committee on Prevention, Detection, Evaluation, and Treatment of High Blood Pressure (JNC 7): is it really practical? Natl Med J India, 2004. 17(4): p. 227. 
58. Maliszewska-Kordybach, B., Sources, Concentrations, Fate and Effects of Polycyclic Aromatic Hydrocarbons (PAHs) in the Environment. Part A: PAHs in Air. Polish Journal Environmental Studies, 1999. 8(3): p. 131-136.

59. Kleiner, H.E., et al., Role of Cytochrome P4501 Family Members in the Metabolic Activation of Polycyclic Aromatic Hydrocarbons in Mouse Epidermis. Chemical Research in Toxicology, 2004. 17(12): p. 1667-1674.

60. Mollerup, S., et al., Sex Differences in Lung CYP1A1 Expression and DNA Adduct Levels among Lung Cancer Patients. Cancer Research, 1999. 59(14): p. 3317-3320.

61. Han, W., et al., Estrogen receptor $\alpha$ increases basal and cigarette smoke extract-induced expression of CYP1A1 and CYP1B1, but not GSTP1, in normal human bronchial epithelial cells. Molecular Carcinogenesis, 2005. 44(3): p. 202-211.

62. Van Winkle, L.S., et al., Gender differences in naphthalene metabolism and naphthalene-induced acute lung injury. American Journal of Physiology - Lung Cellular and Molecular Physiology, 2002. 282(5): p. L1122-L1134.

63. Clark 3rd, J.D., et al., Exposure to polycyclic aromatic hydrocarbons and serum inflammatory markers of cardiovascular disease. Environmental Research, (0).

64. Benowitz, N.L., et al., Cotinine disposition and effects. Clin. Pharm. Ther., 1983. 34(5): p. 604611.

65. Gorber, S.C., et al., The accuracy of self-reported smoking: A systematic review of the relationship between self-reported and cotinine-assessed smoking status. Nicotine \& Tobacco Research, 2009. 11(1): p. 12-24.

66. Perezstable, E.J., N.L. Benowitz, and G. Marin, Is Serum Cotinine a Better Measure of CigaretteSmoking Than Self-Report? Preventive Medicine, 1995. 24(2): p. 171-179.

67. Benowitz, N.L., Cotinine as a Biomarker of Environmental Tobacco Smoke Exposure. Epidemiologic Reviews, 1996. 18(2): p. 188-204.

68. St.Helen, G., et al., Exposure and Kinetics of Polycyclic Aromatic Hydrocarbons (PAHs) in Cigarette Smokers. Chemical Research in Toxicology, 2012. 25(4): p. 952-964.

69. Mcclean, M.D., et al., Using Urinary Biomarkers of Polycyclic Aromatic Compound Exposure to Guide Exposure-Reduction Strategies Among Asphalt Paving Workers. Annals of Occupational Hygiene, 2012. 56(9): p. 1013-1024.

70. Khalil, A., et al., Polycyclic aromatic hydrocarbons potentiate high-fat diet effects on intestinal inflammation. Toxicology Letters, 2010. 196(3): p. 161-167.

71. National Center for Health Statistics. The National Health and Nutrition Examination Survey 2005-2006. Survey Operations Manuals, Brochures, Consent Documents. 2009 [cited 2011 06/25]; Available from: http://www.cdc.gov/nchs/nhanes/nhanes20052006/current nhanes 05 06.htm.

72. Report of the Expert Committee on the Diagnosis and Classification of Diabetes Mellitus. Diabetes Care, 2002. 25(suppl 1): p. s5-s20.

73. Maritim, A.C., R.A. Sanders, and J.B. Watkins, Diabetes, oxidative stress, and antioxidants: A review. Journal of Biochemical and Molecular Toxicology, 2003. 17(1): p. 24-38.

74. Calle, M.C. and M.L. Fernandez, Inflammation and type 2 diabetes. Diabetes Metab, 2012. 38(3): p. 183-91.

75. Bending, D., P. Zaccone, and A. Cooke, Inflammation and type one diabetes. International Immunology, 2012. 24(6): p. 339-346. 
76. Jeffy, B.D., R.B. Chirnomas, and D.F. Romagnolo, Epigenetics of breast cancer: Polycyclic aromatic hydrocarbons as risk factors. Environmental and Molecular Mutagenesis, 2002. 39(23): p. 235-244.

77. Bordeleau, L., et al., Diabetes and breast cancer among women with BRCA1 and BRCA2 mutations. Cancer, 2011. 117(9): p. 1812-1818.

78. Bhatnagar, A., Environmental Cardiology. Circulation Research, 2006. 99(7): p. 692-705.

79. Taylor, A.E., Cardiovascular effects of environmental chemicals. Otolaryngol Head Neck Surg, 1996. 114(2): p. 209-11.

80. Suwa, T., et al., Particulate air pollution induces progression of atherosclerosis. J Am Coll Cardiol, 2002. 39(6): p. 935-42.

81. Binkova, B., et al., DNA adducts and human atherosclerotic lesions. Int J Hyg Environ Health, 2001. 204(1): p. 49-54.

82. De Flora, S., et al., DNA adducts and chronic degenerative disease. Pathogenetic relevance and implications in preventive medicine. Mutat Res, 1996. 366(3): p. 197-238.

83. De Flora, S., et al., Molecular epidemiology of atherosclerosis. FASEB J, 1997. 11(12): p. 1021-31.

84. $\mathrm{Xu}, \mathrm{X}$., et al., Studying associations between urinary metabolites of polycyclic aromatic hydrocarbons (PAHs) and cardiovascular diseases in the United States. Sci Total Environ, 2010. 408(21): p. 4943-8.

85. National Center for Health Statistics. The National Health and Nutrition Examination Survey 2005-2006. Survey Operations Manuals, Brochures, Consent Documents. [cited 2011 06/25]; Available from: http://www.cdc.gov/nchs/nhanes/nhanes20052006/current nhanes 05 06.htm.

86. National Center for Health Statistics. The National Health and Nutrition Examination Survey 2003-2004. Survey Operations Manuals, Brochures, Consent Documents. [cited 2012 06/29]; Available from: http://www.cdc.gov/nchs/nhanes/nhanes2003-2004/nhanes03 04.htm.

87. National Center for Health Statistics. Laboratory procedures used for NHANES 2005-2006. [cited 2012 November,1]; Available from: www.cdc.gov/nchs/nhanes/nhanes2007-2008/lab07 08.

88. CDC, Third National Report on Human Exposure to Environmental Chemicals. 2005, Centers for Disease Control and Prevention. Department of Health and Human Services (DHHS).

89. Vital signs: prevalence, treatment, and control of hypertension--United States, 1999-2002 and 2005-2008. MMWR Morb Mortal Wkly Rep, 2011. 60(4): p. 103-8.

90. Ridker, P.M., S.S. Bassuk, and P.P. Toth, C-reactive protein and risk of cardiovascular disease: evidence and clinical application. Curr Atheroscler Rep, 2003. 5(5): p. 341-9.

91. Danesh, J., et al., Association of fibrinogen, C-reactive protein, albumin, or leukocyte count with coronary heart disease: meta-analyses of prospective studies. JAMA, 1998. 279(18): p. 1477-82.

92. Obana, H., et al., Polycyclic aromatic hydrocarbons in human fat and liver. Bull Environ Contam Toxicol, 1981. 27(1): p. 23-7.

93. Mensah, G.A., et al., Major Coronary Risk Factors and Death from Coronary Heart Disease: Baseline and Follow-up Mortality Data from the Second National Health and Nutrition Examination Survey (NHANES II). American journal of preventive medicine, 2005. 29(5): p. 68-74. 